Article

\title{
Optimal Spectral Combination of a Hyperspectral Camera for Intraoperative Hemodynamic and Metabolic Brain Mapping
}

\author{
Charly Caredda ${ }^{1, *(\mathbb{D})}$, Laurent Mahieu-Williame ${ }^{1} \mathbb{C}$, Raphaël Sablong ${ }^{1}$, Michaël Sdika ${ }^{1}$, \\ Jacques Guyotat ${ }^{2}$ and Bruno Montcel ${ }^{1, *}$ \\ 1 Université de Lyon, INSA-Lyon, Université Claude Bernard Lyon 1, UJM-Saint Etienne, CNRS, Inserm, \\ CREATIS UMR 5220, U1206, F69100 Lyon, France; Laurent.Mahieu-Williame@creatis.insa-lyon.fr (L.M.-W.); \\ Raphael.Sablong@creatis.insa-lyon.fr (R.S.); Michael.Sdika@creatis.insa-lyon.fr (M.S.) \\ 2 Service de Neurochirurgie D, Hospices Civils de Lyon, F69500 Bron, France; jacques.guyotat@chu-lyon.fr \\ * Correspondence: charly.caredda@creatis.insa-lyon.fr (C.C.); bruno.montcel@univ-lyon1.fr (B.M.)
}

Received: 24 June 2020; Accepted: 21 July 2020 ; Published: 27 July 2020

\begin{abstract}
Intraoperative optical imaging is a localization technique for the functional areas of the human brain cortex during neurosurgical procedures. These areas are assessed by monitoring the oxygenated $\left(\mathrm{HbO}_{2}\right)$ and deoxygenated hemoglobin $(\mathrm{Hb})$ concentration changes occurring in the brain. Sometimes, the functional status of the brain is assessed using metabolic biomarkers: the oxidative state of cytochrome-c-oxidase (oxCCO). A setup composed of a white light source and a hyperspectral or a standard RGB camera could be used to identify the functional areas. The choice of the best spectral configuration is still based on an empirical approach. We propose in this study a method to define the optimal spectral combinations of a commercial hyperspectral camera for the computation of hemodynamic and metabolic brain maps. The method is based on a Monte Carlo framework that simulates the acquisition of the intrinsic optical signal following a neuronal activation. The results indicate that the optimal spectral combination of a hyperspectral camera aims to accurately quantify the $\mathrm{HbO}_{2}(0.5 \%$ error $), \mathrm{Hb}$ ( $4.4 \%$ error), and oxCCO ( $15 \%$ error) responses in the brain following neuronal activation. We also show that RGB imaging is a low cost and accurate solution to compute $\mathrm{Hb}$ maps ( $4 \%$ error), but not accurate to compute $\mathrm{HbO}_{2}$ ( $48 \%$ error) or oxCCO (1036\% error) maps.
\end{abstract}

Keywords: hemodynamic brain mapping; metabolic brain mapping; Monte Carlo simulations; intraoperative imaging; optical imaging; hyperspectral imaging; RGB imaging

\section{Introduction}

Non-invasive functional brain mapping is an imaging technique used to localize the functional areas of the patient brain. This technique is used during brain tumor resection surgery to indicate to the neurosurgeon the cortical tissues that should not be removed without cognitive impairment. Functional magnetic resonance imaging (fMRI) [1] is the preoperative gold standard for the identification of the patient brain functional areas. However, after patient craniotomy, a brain shift invalidates the relevance of neuro-navigation to intraoperatively localize the functional areas of the patient brain [2]. In order to prevent any localization error, intraoperative MRI has been suggested, but it complicates the surgery gesture, which makes it rarely used. For these reasons, electrical brain stimulation [3] is preferred during neurosurgery. However, this technique suffers from limitations because the measurements could trigger epilepsy seizures. Since optical imaging combined with a quantitative modeling of brain hemodynamic biomarkers could evaluate in real time the functional areas during neurosurgery [4-6], this technique could serve as a tool of choice to complement the electrical brain stimulation. 
Hyperspectral imaging allows the in vivo monitoring of the hemodynamic and metabolic status of an exposed cortex. Hyperspectral imaging provides spatially and spectrally resolved images using numerous and contiguous spectral bands [7]. In comparison, a standard color camera (or RGB camera) acquires three colors (red, green, and blue) using broad and overlapping spectral detectors. Both techniques have the ability to measure the oxygenation changes in the tissue using the modified Beer-Lambert law [5,8-12]. In functional brain mapping studies, the concentration changes of oxy- $\left(\Delta \mathrm{C}_{\mathrm{HbO}_{2}}\right)$ and deoxy-hemoglobin $\left(\Delta \mathrm{C}_{\mathrm{Hb}}\right)$ can be analyzed to identify the activated cortical areas [5,13-21]. The acquisition of the intrinsic signal in the near-infrared range offers the potential to monitor the brain metabolism with the quantification of the concentration changes of the oxidative state of cytochrome-c-oxidase $\left(\Delta C_{o x C C O}\right)$ [22-25]. Hyperspectral and color cameras combined with a white light illumination are simple and powerful tools for the computation of intraoperative functional brain maps. The objective is to guide the neurosurgeon during brain surgery to prevent any functional impairments after surgical procedures (tumor resection).

In the literature, all wavelength bands acquired by hyperspectral imaging setups are used to measure the hemodynamic and metabolic changes in the brain [10,24-26]. However, there are some studies in which the choice of the selected spectral bands is discussed. Bale et al. [23] showed that tens to hundreds of spectral bands acquired with a broadband near-infrared spectroscopy setup $(780 \mathrm{~nm}$ to $900 \mathrm{~nm})$ can be used to measure the oxCCO concentration changes. Arifler et al. [27] showed that eight wavelength combinations between $780 \mathrm{~nm}$ and $900 \mathrm{~nm}$ give rise to the least possible estimation errors for the deconvolution of $\Delta C_{\mathrm{HbO}_{2}}, \Delta C_{\mathrm{Hb}}$, and $\Delta C_{o x C C O}$ when compared to a gold standard (121 wavelengths included between $780 \mathrm{~nm}$ and $900 \mathrm{~nm}$ ). Giannoni et al. [28] proposed a Monte Carlo framework to investigate the performances of broadband spectroscopy to quantify the brain hemodynamic and metabolic responses. The results of this study indicated that eight wavelength between $780 \mathrm{~nm}$ and $900 \mathrm{~nm}$ should be selected to provide minimal differences in quantification compared to a gold standard of 121 wavelengths $(780 \mathrm{~nm}$ to $900 \mathrm{~nm})$. Sudakou et al. [29] proposed a method based on the error propagation analysis and Monte Carlo simulations (three layer model: scalp, skull, and brain) allowing the estimation of the cytochrome-c-oxidase uncertainty in data measured with a multispectral time-resolved near-infrared spectroscopy device. The results of this study indicated that 16 wavelengths between 688 and $875 \mathrm{~nm}$ could be used to minimize the standard deviation of the cytochrome-c-oxidase concentration changes in the brain layer. Wavelength optimization problems have also been studied for other optical imaging techniques such as near-infrared optical tomography. Chen et al. [30] identified seven laser diodes among 38 commercially available diodes in the range of $633-980 \mathrm{~nm}$ to estimate four chromophores $\left(\mathrm{HbO}_{2}, \mathrm{Hb}\right.$, water, and lipids) and the scattering prefactor in breast tissue. Chen et al. used the condition number and the residual norm to identify the optimal matrix used for the resolution of a linear system and thus to estimate four chromophores and the scattering prefactor in breast tissue. The optimal wavelengths were identified for a large residual norm and small condition number. The residual norm and the condition number can be interpreted as parameters representing the uniqueness and stability of the solution, respectively.

The commercial hyperspectral cameras have limited choices in the available spectral bands and do not have a spectral resolution as high as the broadband spectroscopy devices used by Bale et al. and Arifler et al. Therefore, the optimal spectral bands identified in these studies may not be available with the commercial cameras. Moreover, the more spectral bands are used, the more time is needed to compute functional brain maps. Since time is the key factor in intraoperative imaging, the smallest number of spectral bands must be acquired while ensuring minimal quantification errors.

First, we propose in this study a method to define the optimal spectral combinations of a commercial hyperspectral camera for intraoperative hemodynamic and metabolic brain mapping. This method could be used with any hyperspectral or standard RGB camera to evaluate its ability to compute accurate hemodynamic $\left(\Delta C_{\mathrm{HbO}_{2}}\right.$ and $\left.\Delta C_{\mathrm{Hb}}\right)$ and/or metabolic $\left(\Delta C_{o x C C O}\right)$ brain maps following neuronal activation. The method is based on the Monte Carlo simulations of the acquisition 
of the intrinsic signal acquired by a camera. All spectral combinations of the hyperspectral camera are tested to evaluate the optimal spectral configuration that minimize the quantification errors in $\Delta C_{\mathrm{HbO}_{2}}, \Delta C_{\mathrm{Hb}}$, and $\Delta C_{o x C C O}$. In this work, we also show that a spectral correction [10] of the reflection spectra acquired by a mosaic hyperspectral sensor is mandatory to minimize the chromophores' quantification errors. Finally, we compare standard RGB imaging and hyperspectral imaging for hemodynamic and metabolic brain mapping. We demonstrate that RGB imaging is a low cost, but not an accurate solution to identify the functional areas in a patient brain based on the analysis of the cortical hemodynamics. Hyperspectral imaging is the ideal solution for an accurate computation of hemodynamic and metabolic brain maps.

\section{Materials and Methods}

We simulated the acquisition of the intrinsic reflection spectra of a patient exposed cortex using hyperspectral imaging to determine the optimal spectral configuration for the computation of hemodynamic and/or metabolic brain maps. We also simulated the acquisition of the intrinsic reflection spectra based on standard RGB imaging to evaluate the potential benefit of using hyperspectral imaging.

\subsection{Simulated Setup}

In this study, the imaging system represented in Figure 1 was simulated. This setup was used in our previous study [5] for the identification of functional areas based on RGB imaging. A continuous wave white light source illuminated the patient exposed cortex. The light source was a halogen bulb (OSRAM Classic 116W 230V) whose spectra was measured with a USB 2000 spectrometer [31]. The intrinsic reflection spectra were acquired by a hyperspectral camera (XIMEA MQ022HG-IM-SM5X5-NIR) and standard RGB camera (BASLER acA2000-165uc). Both RGB and hyperspectral cameras acquire spectrally and spatially resolved images, but these two systems differ in their spectral resolution. The RGB camera acquires three broad and overlapping spectra over $300 \mathrm{~nm}$ to $700 \mathrm{~nm}$ using a Bayer filter [32] mounted on a CMOS sensor. The hyperspectral camera acquires 25 narrow and contiguous spectral bands in the red in the near-infrared range. A $5 \times 5$ mosaic filter (25 Fabry-Perot filters) mounted on a CMOS sensor was used for the acquisition of the 25 spectral bands. Each Fabry-Perot filter has two transmission peaks. The first or the second peak can be selected by mounting an interference filter on the camera lens. In our study, a long pass filter mounted on the hyperspectral camera lens selected the wavelength included between $675 \mathrm{~nm}$ and $975 \mathrm{~nm}$.

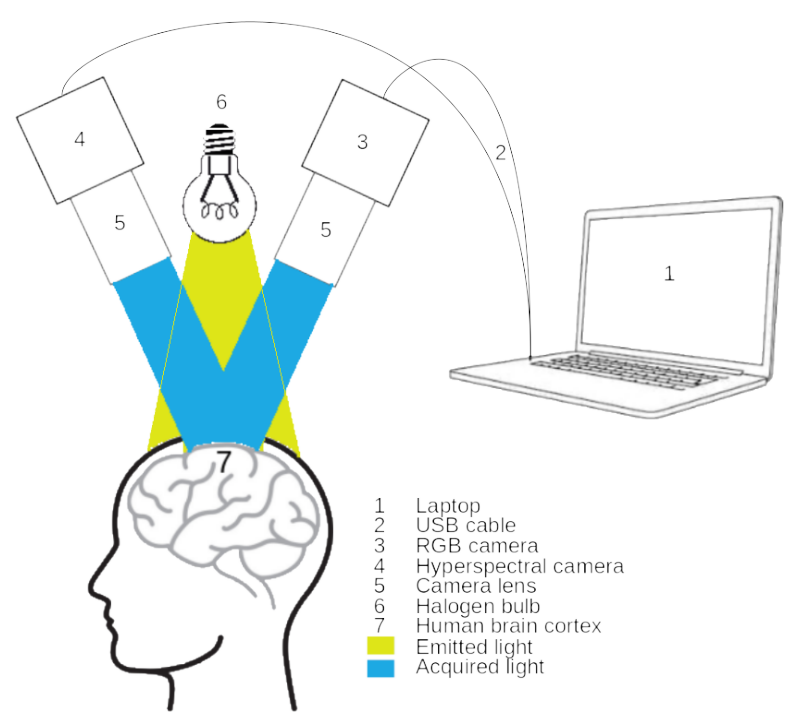

Figure 1. Schematic of the simulated imaging system. 
The intensity $R$ measured by the spectral channel $k$ of the camera $(k \in[1 ; 3]$ for RGB imaging and $k \in[1 ; 25]$ for hyperspectral imaging) is expressed by the following equation:

$$
R_{k}=\int_{\lambda_{\min }}^{\lambda_{\max }} D_{k}(\lambda) \cdot \Phi(\lambda) \cdot d \lambda
$$

The integral runs from $\lambda_{\min }=400 \mathrm{~nm}$ to $\lambda_{\max }=700 \mathrm{~nm}$ for RGB imaging and from $\lambda_{\min }=675 \mathrm{~nm}$ to $\lambda_{\max }=975 \mathrm{~nm}$ for hyperspectral imaging. These wavelengths delimit the ranges of the spectral sensitivity profiles provided by the camera manufacturers. $D_{k}$ is the spectral sensitivity of spectral channel $k$ of the camera, provided by the camera manufacturer. $\Phi$ is the diffuse reflection spectra of the illuminated tissue.

\subsection{Hyperspectral Correction}

In hyperspectral imaging based on mosaic filters, the use of the interference filter does not completely eliminate the crosstalk between the camera spectral sensitivities. To approach the ideal spectral sensitivity $D^{\text {ideal }}$ (provided by the camera manufacturer), Pichette et al. [10] proposed to compute a linear combination of the spectral sensitivities:

$$
D_{m}^{\text {Corr }}(\lambda)=\sum_{k=1}^{25} X_{m, k} \cdot D_{k}(\lambda)
$$

with $D_{m}^{\text {Corr }}$ the corrected spectral sensibility of the spectral channel $m(m \in[1 ; 25])$ and $X$ the $25 \times 25$ correction matrix. This correction matrix $X$ was computed with a linear least squares fitting:

$$
\min _{X}\left\|D^{\text {ideal }}-X . D\right\|_{2}^{2},
$$

The normalized spectral sensitivities $\left(\int D(\lambda) \cdot d \lambda=1\right)$ are represented in Figure 2.
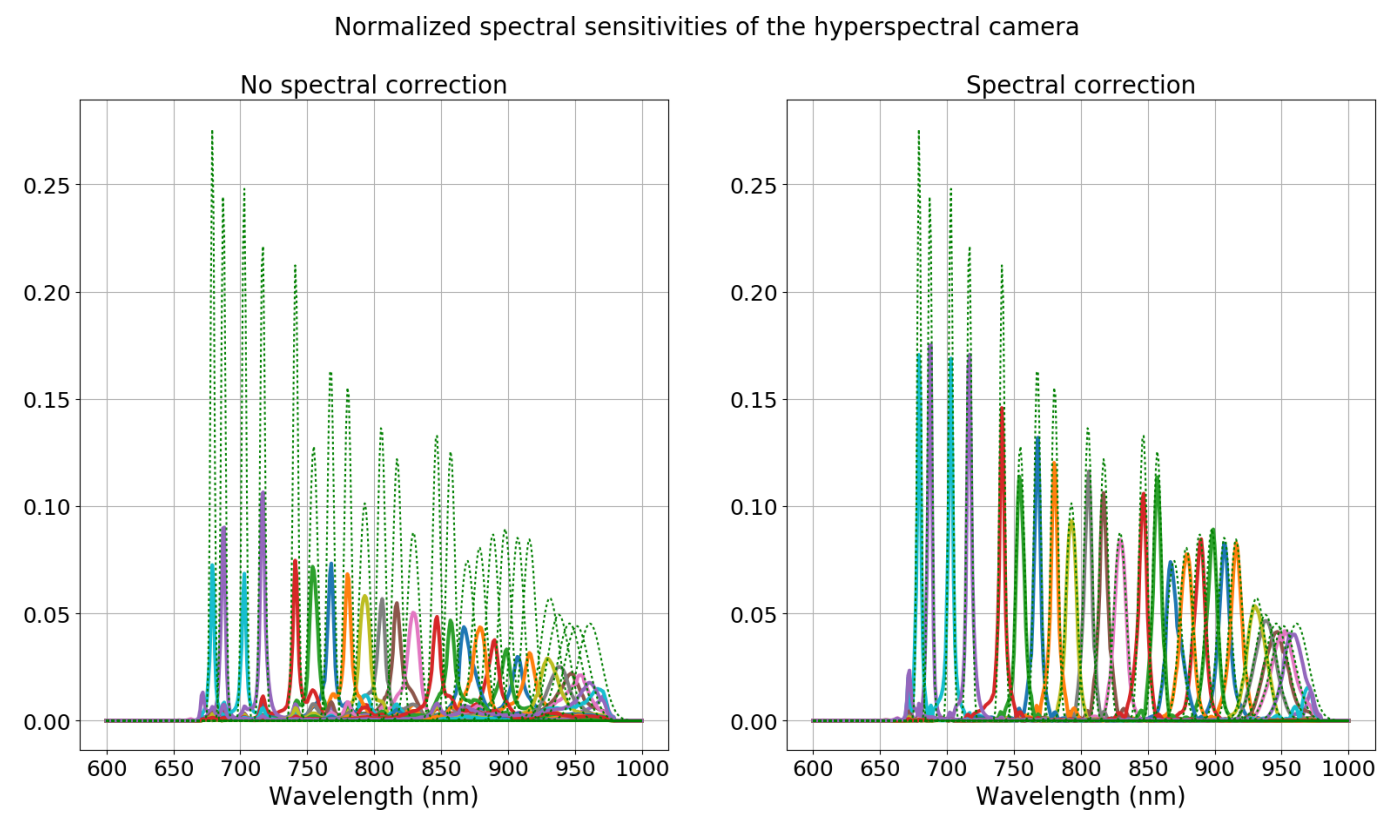

Figure 2. Spectral sensitivities of the hyperspectral camera XIMEA MQ022HG-IM-SM5X5-NIR. The uncorrected spectral sensitivities are plotted in solid lines on the left side of the figure and the corrected ones on the right side. The ideal spectral sensitivities are plotted in green dashed lines.

As the normalized spectral sensitivity $\left(\int D(\lambda) \cdot d \lambda=1\right)$ represents a probability density function, the Kullback-Leibler divergence can be used to quantify the performance of the spectral correction [33]: 


$$
K L_{k}\left(D_{k} \| D_{k}^{\text {ideal }}\right)=\int_{\lambda_{\min }}^{\lambda_{\max }} D_{k}(\lambda) \cdot \log \left(\frac{D_{k}(\lambda)}{D_{k}^{\text {ideal }}(\lambda)}\right) d \lambda .
$$

$K L_{k}\left(D_{k} \| D_{k}^{i d e a l}\right)$ represents the Kullback-Leibler divergence (KL divergence) computed between the spectral sensitivity of the channel $k$ (corrected or uncorrected) and the ideal spectral sensitivity of the channel $k$. The $K L$ divergence represents a measure of dissimilarity between $D_{k}$ and $D_{k}^{\text {ideal }}$. A value equal to 0 indicates the equality of the two probability density functions. The $K L$ divergence values computed for the corrected and uncorrected spectral sensitivities are represented in Figure 3.

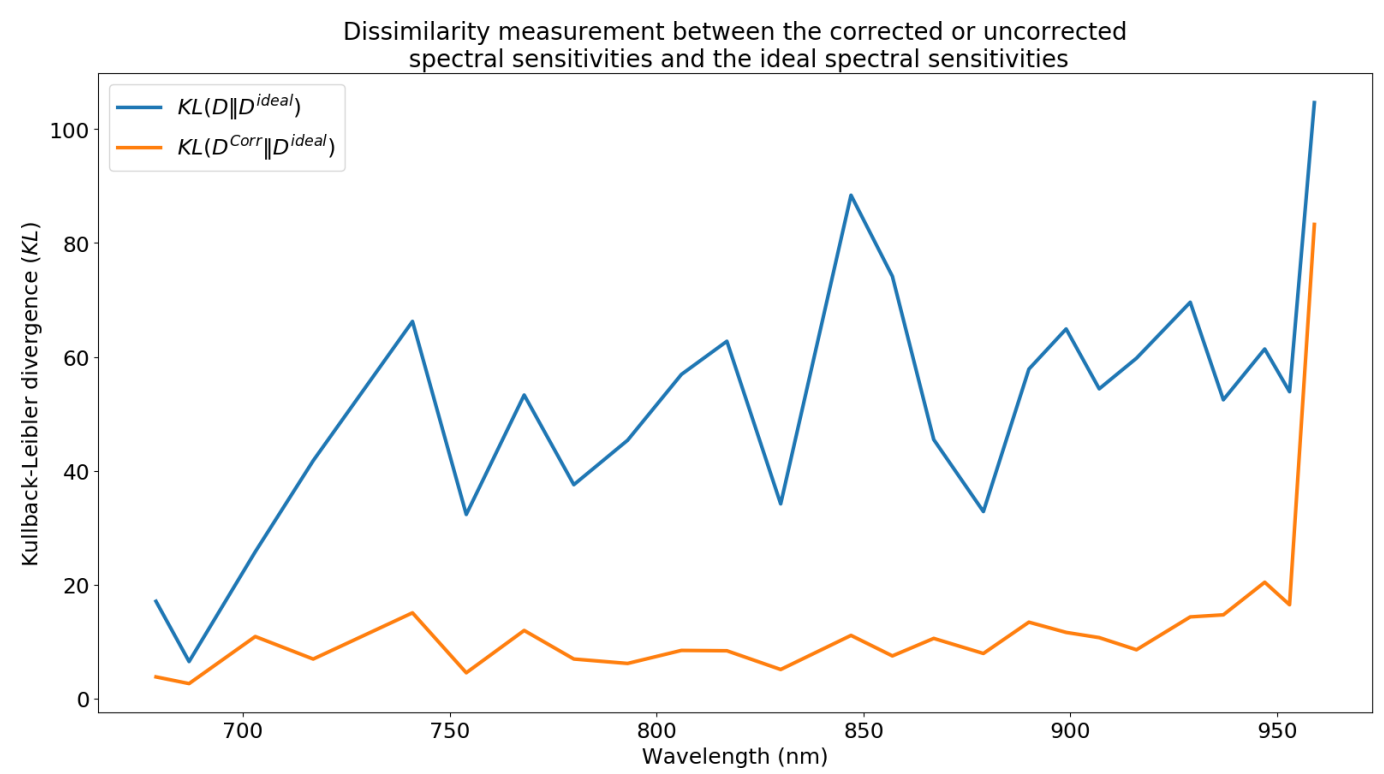

Figure 3. Measure of dissimilarities between the corrected (in orange) or uncorrected (in blue) spectral sensibilities and the ideal spectral sensitivities.

The $K L$ divergence computed between the uncorrected spectral sensitivities and the ideal spectral sensitivities is on average four times higher than those computed between the corrected spectral sensitivities and the ideal spectral sensitivities.

\subsection{Sensor Signal-to-Noise Ratio}

The signal-to-noise ratios (SNR) of the spectral channel $k$ of the RGB and hyperspectral cameras were measured by acquiring the white light reflected at the surface of a calibration target (plain white sheet of paper) during two minutes with an integration time set to $33 \mathrm{~ms}$ :

$$
\operatorname{SNR}(k)=\frac{\mu_{k}}{\sigma_{k}} .
$$

$\mu_{k}$ denotes the spectral channel $k$ mean value obtained by computing the mean value of the temporal intensity averaged over the sensor area. $\sigma_{k}$ is the spectral channel $k$ standard deviation value obtained by computing the standard deviation value of the temporal intensity averaged over the sensor area. The illumination source power, quantum efficiency and integration time of the cameras directly impact the SNR values. The SNR values of the RGB and hyperspectral cameras we used in this study are represented in Figure 4. The SNR of the red channel of the RGB camera is 1.1 times greater than the one of the green channel and 1.4 times greater than the one of the blue channel. The mean value of the SNR of the hyperspectral is 3.9 times lower than the SNR value of the red channel of the RGB camera. Note that these values are dependent on our experimental configuration and directly impact the amount of noise and the quantification error in the simulated concentration changes. 


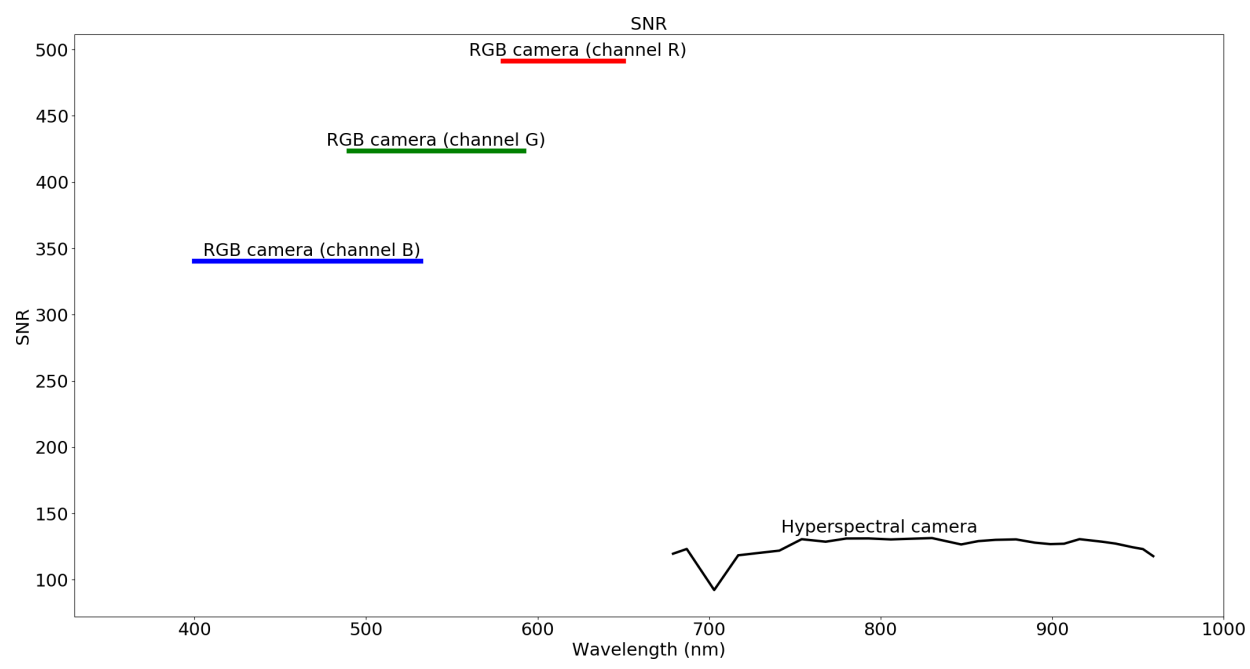

Figure 4. SNR values of each spectral channel of the RGB and hyperspectral cameras.

\subsection{Simulation of the Patient Cortical Activation}

Following the patient physiological activity, a hemodynamic change occurs in the activated cortical areas with an increase of the oxygenated hemoglobin concentration $\left(\mathrm{C}_{\mathrm{HbO}_{2}}\right)$ and a decrease of the deoxygenated hemoglobin concentration changes $\left(\mathrm{C}_{\mathrm{Hb}}\right)$. These hemodynamic changes are not the only ones that occur during a patient physiological activity. The concentration of the oxidative state of cytochrome-c-oxidase $\left(C_{o x C C O}\right)$ also varies [22]. Cytochrome-c-oxidase (CCO) is an enzyme in the mitochondria that is involved in the aerobic metabolism of glucose. The total concentration of CCO does not change over a short time period (in the order of hours). However, it is possible to assess the differences between the oxidized and reduced states of $\mathrm{CCO}$ to obtain an indicator of the change in the CCO redox state.

The patient cortical activation was simulated by Monte Carlo simulations [34]. A volume of $60 \mathrm{~mm} \times 60 \mathrm{~mm} \times 60 \mathrm{~mm}$ of grey matter was modeled under a homogeneous white light illumination. Each voxel of the modeled tissue included the information of optical parameters (absorption $\mu_{a}$, scattering $\mu_{s}$, anisotropy $g$ coefficients, and refractive index $n$ ). A white light illumination was simulated by scanning the optical parameters along the entire illumination spectrum (from $400 \mathrm{~nm}$ to $1000 \mathrm{~nm}$ in steps of $1 \mathrm{~nm}$ ). The size of the modeled tissues was chosen in accordance with the photon sensitivity profile $[5,35]$ computed for the detector situated at the center of the top face of the volumes. To avoid any photon loss and inexact results due to the boundary conditions (the simulation of the travel of a packet of photon stops when this packet of photon leaves the volume), the size of the models was set to $60 \times 60 \times 60$ voxels with a resolution of $1 \mathrm{~mm}^{3}$. The $10^{8}$ packets of photons were homogeneously illuminating the modeled surface. The optical mean path length and the diffuse reflection spectra were measured at the detector position with an integration time set to $33 \mathrm{~ms}$, and the diffuse reflection spectra was normalized to a unitary source.

The optical parameters were taken from the literature and correspond to a nominal physiological condition [36-41]. The anisotropy, reduced scattering coefficients, and the refractive index used in the Monte Carlo simulations are summarized in Table 1 . The scattering coefficient $\mu_{s}$ was calculated from the reduced scattering coefficient $\left(\mu_{s}^{\prime}\right)$ and the anisotropy coefficient $(g): \mu_{s}=\mu_{s}^{\prime} /(1-g)$. In Table 1 , $\lambda$ denotes the wavelength dependence of the reduced scattering coefficient (in $\mathrm{nm}$ ).

Table 1. Anisotropy, reduced scattering coefficients, and refractive index used in the Monte Carlo simulations.

\begin{tabular}{ccc}
\hline Anisotropy Coefficient $\boldsymbol{g}$ & Reduced Scattering Coefficient $\boldsymbol{\mu}_{\boldsymbol{s}}^{\mathbf{(}\left(\mathbf{c m}^{-\mathbf{1}}\right)}$ & Refractive Index $\boldsymbol{n}$ \\
\hline $0.85[42]$ & $40.8 \cdot{\frac{\lambda^{500}}{}}^{-3.089}[39]$ & $1.36[38]$ \\
\hline
\end{tabular}


The absorption coefficients were calculated using the chemical composition of the chromophores of the simulated tissues, which are summarized in Table 2.

Table 2. Chemical composition of the chromophores of the simulated volumes of grey matter.

\begin{tabular}{ccc}
\hline & Non-Activated Grey Matter & Activated Grey Matter \\
\hline Water & $73 \%$ & $73 \%$ \\
$C_{\mathrm{Hb}}\left(\mu \mathrm{mol} \cdot \mathrm{L}^{-1}\right)$ & 22.1 & 18.35 \\
$C_{\mathrm{HbO}}\left(\mu \mathrm{mol} \cdot \mathrm{L}^{-1}\right)$ & 65.1 & 70.1 \\
$C_{o x C C O}\left(\mu \mathrm{mol} \cdot \mathrm{L}^{-1}\right)$ & 5.3 & 5.8 \\
\hline
\end{tabular}

The chemical differences between the activated and non-activated grey matter are represented by a $5 \mu \mathrm{mol} \cdot \mathrm{L}^{-1}$ increase of $C_{\mathrm{HbO}_{2}}$, a $3.75 \mu \mathrm{mol} \cdot \mathrm{L}^{-1}$ decrease of $C_{\mathrm{Hb}}$, and a $0.5 \mu \mathrm{mol} \cdot \mathrm{L}^{-1}$ increase of $\mathrm{C}_{\text {oxCCOO}}$. These concentration changes are consistent with those defined in the literature. The reflection spectra and the optical mean path length were measured at the center of the top face of the volume.

\subsection{Modified Beer-Lambert Law}

The modified Beer-Lambert law is used to convert the acquired intrinsic reflection spectra into concentration changes. Optical functional brain maps are computed by assessing the oxyand deoxy-genated hemoglobin concentration changes $\left(\Delta C_{\mathrm{HbO}_{2}}\right.$ and $\left.\Delta C_{\mathrm{Hb}}\right)[9,10,43]$. Using the near-infrared range, the oxidative state of cytochrome-c-oxidase concentration changes $\left(\Delta C_{o x C C O}\right)$ can also be quantified [23]. The modified Beer-Lambert law can be expressed as a linear system [9]:

$$
\left[\begin{array}{c}
\Delta A_{1} \\
\vdots \\
\Delta A_{N}
\end{array}\right]=\left[\begin{array}{ccc}
E_{1, \mathrm{HbO}_{2}} & E_{1, \mathrm{Hb}} & E_{1, o x \mathrm{CCO}} \\
\vdots & \vdots & \vdots \\
E_{\mathrm{N}, \mathrm{HbO}_{2}} & E_{N, \mathrm{Hb}} & E_{N, o x \mathrm{CCO}}
\end{array}\right] \times\left[\begin{array}{c}
\Delta C_{\mathrm{HbO}_{2}} \\
\Delta C_{\mathrm{Hb}} \\
\Delta C_{o x \mathrm{CCO}}
\end{array}\right]
$$

with

$$
E_{k, n}=\int \epsilon_{n}(\lambda) \cdot D_{k}(\lambda) \cdot S(\lambda) \cdot L(\lambda) \cdot d \lambda .
$$

$\Delta A_{k}$ is the absorbance changes measured by the spectral channel $k$ of the camera $(k \in[1 ; N]$ with $N \in[3 ; 25]$ for hyperspectral imaging and $k \in[1 ; 3]$ for RGB imaging):

$$
\Delta A_{k}=\log _{10}\left(\frac{R_{k}^{G M_{1}}}{R_{k}^{G M_{2}}}\right) .
$$

$R_{k}^{G M_{1}}$ is the intensity of the non-activated grey matter (see Section 2.4) acquired by the spectral channel $k$ of the camera. $R_{k}^{G M_{2}}$ is the intensity of the activated grey matter (see Section 2.4) acquired by the spectral channel $k$ of the camera. The intensities were calculated with Equation (1) using the reflection spectra simulated by Monte Carlo simulations. $\Delta C_{H b}$ is the deoxygenated hemoglobin molar concentration changes (in mol. $\mathrm{L}^{-1}$ ). $\Delta \mathrm{C}_{\mathrm{HbO}_{2}}$ is the oxygenated hemoglobin molar concentration changes (in mol $\cdot \mathrm{L}^{-1}$ ) and $\Delta \mathrm{C}_{o x C C O}$ the oxidative state of cytochrome-c-oxidase concentration changes (in $\mathrm{mol} \cdot \mathrm{L}^{-1}$ ). $\epsilon_{n}$ is the extinction coefficient of the chromophore $n$ (in $\mathrm{L} \cdot \mathrm{mol}^{-1} \cdot \mathrm{cm}^{-1}$ ). The spectral sensitivity of the channel $k$ is represented by $D_{k}(\lambda)$, and $S(\lambda)$ is the normalized intensity spectrum of the light source. $L(\lambda)$ is the wavelength dependent mean optical path length of the photons traveling in tissue estimated by Monte Carlo simulations (in $\mathrm{cm}$ ); see Section 2.4. The concentration changes were obtained by matrix inversion once the matrix $E$ was calculated.

\subsection{Determination of the Optimal Spectral Configuration of the Hyperspectral Camera}

The quantification of $\Delta C_{\mathrm{Hb}}, \Delta C_{\mathrm{HbO}_{2}}$, and $\Delta C_{o x C C O}$ can be achieved using at least three wavelengths [44]. With our hyperspectral camera, twenty-five spectral bands were acquired. 
Therefore, we can then ask ourselves what is the optimal spectral configuration for the assessment of $\Delta C_{\mathrm{Hb}}, \Delta C_{\mathrm{HbO}_{2}}$, and $\Delta C_{o x C C O}$ ? If $N$ spectral bands were used $(N \in[3 ; 25]), P$ combinations may be investigated:

$$
\forall N \in[3 ; 25] \quad P=\frac{25 !}{(25-N) ! \times N !}
$$

\subsubsection{Noise in Simulations}

In order to evaluate the robustness of the chromophores' quantification, zero mean Gaussian noises were added to the simulated quantities. The Monte Carlo noises (noise on diffuse reflection spectra $\phi$ and mean path length $L$ ) were estimated for each simulated wavelength by launching the MCX software $10^{3}$ times using random seeds. The seed is a number used to generate a random number with a pseudorandom number generator. For each wavelength, the standard deviations of the Monte Carlo noises were measured on these $10^{3}$ simulations. The Monte Carlo noise depends on the number of packets of photons used in the simulation. In addition, the noise of the hyperspectral and RGB cameras that were experimentally measured (see Section 2.3) were added to the simulated intensities.

\subsubsection{Simulation Based Method}

For all the possible $P$ combinations for a group of $N$ spectral bands (see Equation (9)), the quantification errors in $\Delta C_{\mathrm{Hb}}, \Delta C_{\mathrm{HbO}_{2}}$, or $\Delta C_{o x C C O}$ were computed:

$$
E_{\Delta C_{n}}(N, p)=\left|\frac{\Delta C_{n}^{\text {Expected }}-\Delta C_{n}^{\text {Estimated }}(N, p)}{\Delta C_{n}^{\text {Expected }}}\right| \times 100 .
$$

Note that this quantification error is represented in absolute values. $\Delta C_{n}^{\text {Expected }}$ is the concentration changes of the chromophore $n$ that were expected to be measured in the tissue; see Section 2.4. $\Delta C_{n}^{\text {Estimated }}(N, p)$ is the concentration changes of the chromophore $n$ estimated from the simulated data using the combination $p(p \in[1 ; P])$ for a group of $N$ spectral bands; see Equation (9). The computation of $\Delta C_{n}^{\text {Estimated }}(N, p)$ and $E_{\Delta C_{n}}(N, p)$ was repeated $10^{3}$ times to get their mean and the standard deviation values. Different noises were added to the simulated reflection spectra $\phi$, mean path length $L$, and camera intensities $R$ for each iteration. For each group of $N$ spectral bands, the optimal spectral combination among the $P$ possibilities was determined using Equation (11):

$$
\min _{p}\left(\sqrt{m\left(E_{\Delta C_{n}}(N, p)\right)^{2}+\sigma\left(E_{\Delta C_{n}}(N, p)\right)^{2}}\right) .
$$

$m$ and $\sigma$ designate the mean and standard deviation functions, respectively. This metric was chosen to identify the spectral combination of $N$ spectral bands that minimize the average quantification errors while ensuring the reproduction of measurements. Equation (11) leads to the determination of the combination $p_{n}^{N}$, which is the best combination of $N$ spectral bands for the deconvolution of the chromophore $n$. Finally, the optimal spectral configurations of the hyperspectral camera for the quantification of $\Delta C_{\mathrm{HbO}_{2}}, \Delta C_{\mathrm{Hb}}$, and $\Delta C_{o x C C O}$ were obtained using Equation (12):

$$
\min _{p_{n}^{N}}\left(\sqrt{m\left(E_{\Delta C_{n}}\left(N, p_{n}^{N}\right)\right)^{2}+\sigma\left(E_{\Delta C_{n}}\left(N, p_{n}^{N}\right)\right)^{2}}\right) .
$$

Note that the spectral configurations determined by Equation (12) are not necessarily the same for the deconvolution of $\Delta C_{\mathrm{HbO}_{2}}, \Delta C_{\mathrm{Hb}}$, and $\Delta C_{o x C C O}$. In other words, this means that three distinct configurations may be used. 


\subsection{Application to a Functional Brain Mapping Study}

In functional brain mapping studies, the measured hemodynamic concentration changes' time courses are compared to the patient theoretical hemodynamic responses. These theoretical responses are obtained by convolving the hemodynamic impulse response function to the window function representing the patient physiological stimulus. The functional areas may be identified by testing the Pearson correlation coefficients (computed between the concentration changes' time courses and the theoretical hemodynamic responses) and the concentration changes averaged over the patient activity period with several $T$-tests $[5,25]$. Another technique derived by BOLD fMRI (blood oxygenation level dependent, functional magnetic resonance imaging) is the NIRS SPM (near-infrared spectroscopy statistical parametric mapping) analyses $[13,14]$ that use a general linear model and random field theory to identify the functional areas in a pixel-wise manner.

We built a simulation of the grey matter hemodynamic and metabolic responses following a patient physiological stimulus based on the Monte Carlo framework described in Section 2.4. The intrinsic signal was acquired during $60 \mathrm{~s}$ at 0.5 frames per second with the integration time set to $33 \mathrm{~ms}$. The following paradigm was simulated: $18 \mathrm{~s}$ of rest, $20 \mathrm{~s}$ of neuronal stimulation, followed by $22 \mathrm{~s}$ of rest. We incorporated the temporal hemodynamic response, as well as the temporal oxCCO response following neuronal activation. The hemodynamic responses were obtained by convolving the hemodynamic impulse response function to the window function representing the patient physiological stimulus. The oxCCO response function was introduced by Wobst et al. [45] to describe the metabolic response in the primary and adjacent visual cortex for a stimulus of 6 to $24 \mathrm{~s}$. The theoretical time courses can be roughly represented by the function $h(t)=a \cdot t^{2} \cdot e^{-t}$ convolved with the window function representing the patient physiological stimulus. In this function, $t$ represents the time (in s) and $a$ is the characteristic amplitude of $\Delta C_{o x C C O}$. The theoretical temporal variation of the $\Delta C_{\mathrm{HbO}_{2}}, \Delta C_{\mathrm{Hb}}$, and $\Delta C_{o x C C O}$ values are represented in Figure 5. Note that these values varied homogeneously in the modeled tissue. During these absorption perturbations, the other optical coefficients were kept constant.

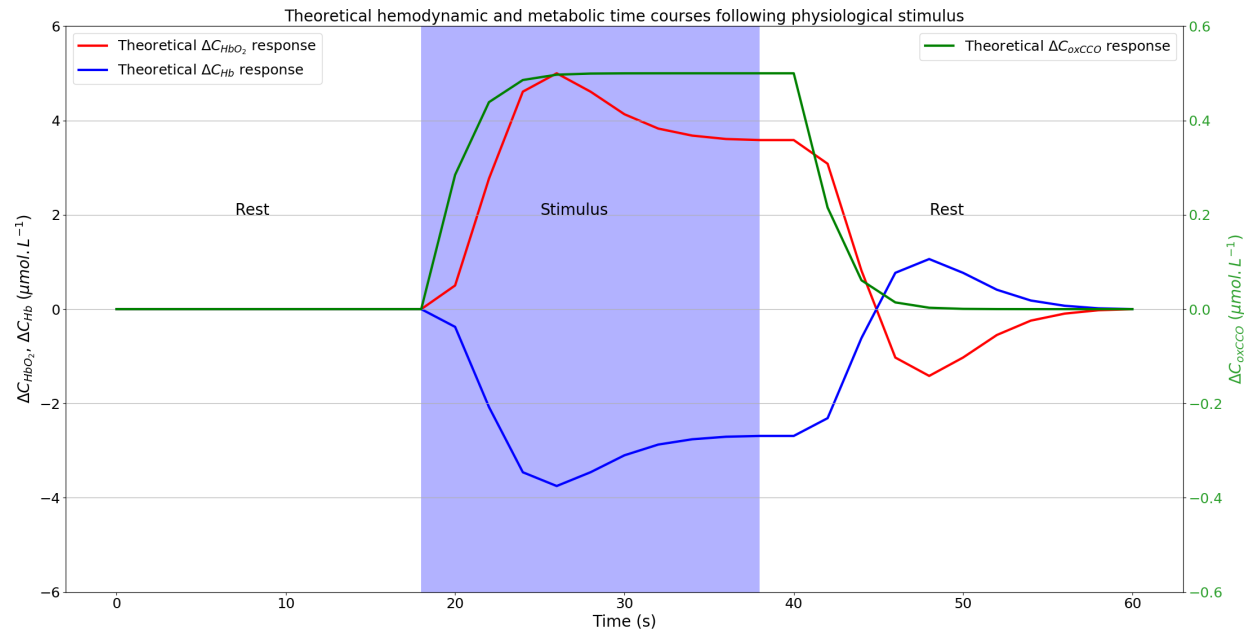

Figure 5. Theoretical $\Delta C_{\mathrm{HbO}_{2}}$ (in red), $\Delta C_{\mathrm{Hb}}$ (in blue), and $\Delta \mathrm{C}_{o x C C O}$ (in green) responses following the patient physiological activity. $\Delta \mathrm{C}_{\mathrm{HbO}_{2}}$ and $\Delta C_{\mathrm{Hb}}$ are expressed by the left vertical axis. $\Delta \mathrm{C}_{\mathrm{oxCCO}}$ is expressed by the right vertical axis. The blue rectangle represent the "patient" physiological stimulus.

With these simulations, we can evaluate the potential benefit from using hyperspectral imaging for the computation of hemodynamic brain maps instead of using a standard RGB camera (see our previous studies $[5,11,12])$. This approach aims to simulate the quantitative values that are computed in clinical functional brain mapping applications. This makes it possible to test the influence of the optimal spectral band configuration on the quantities measured in clinical applications. For this purpose, the Pearson correlation coefficient is computed. The correlation coefficient was calculated between the 
theoretical hemodynamic time courses (see Figure 5) and the hemodynamic time courses simulated with the optimal spectral configuration of the hyperspectral camera (see Section 2.6). The correlation coefficient was also computed with the data simulated with RGB imaging. We can also investigate the ability of hyperspectral imaging to monitor the brain metabolism. For this purpose, the Pearson correlation coefficient values were computed between the theoretical oxCCO time course (see Figure 5) and the $\Delta C_{o x C C O}$ time courses simulated with the optimal spectral configuration of the hyperspectral camera (see Section 2.6).

We also compared our results with the measurements computed with the gold standard of 121 wavelengths included between $780 \mathrm{~nm}$ and $900 \mathrm{~nm}$ [23] and with the 8 wavelengths identified by Arifler et al. [27]. For this purpose, we simulated the hemodynamic and/or metabolic monitoring using two or three chromophore systems that used these wavelengths. Note that we did not incorporate noises in these simulations of references.

\section{Results}

\subsection{Determination of the Optimal Spectral Configuration of the Hyperspectral Camera}

In Figure 6, the quantification errors in $\Delta C_{\mathrm{HbO}_{2}}, \Delta C_{\mathrm{Hb}}$ and $\Delta C_{o x C C O}$ (see Equation (10)) obtained for the best simulation-based deconvolution systems of $N$ spectral bands $(N \in[3 ; 25])$ are represented. The solid lines stand for the mean quantification error. The colored areas represent the dispersion range of the quantification errors. The vertical dashed lines indicate the optimal number of spectral bands for the deconvolution of each chromophore. Both corrected (in green) and uncorrected spectral configuration (in red) are represented. Note that these data were obtained using noisy measurements; zeros mean Gaussian noises were added to the camera intensities and to the Monte Carlo quantities (mean path length and reflection spectra); see Section 2.6.1.
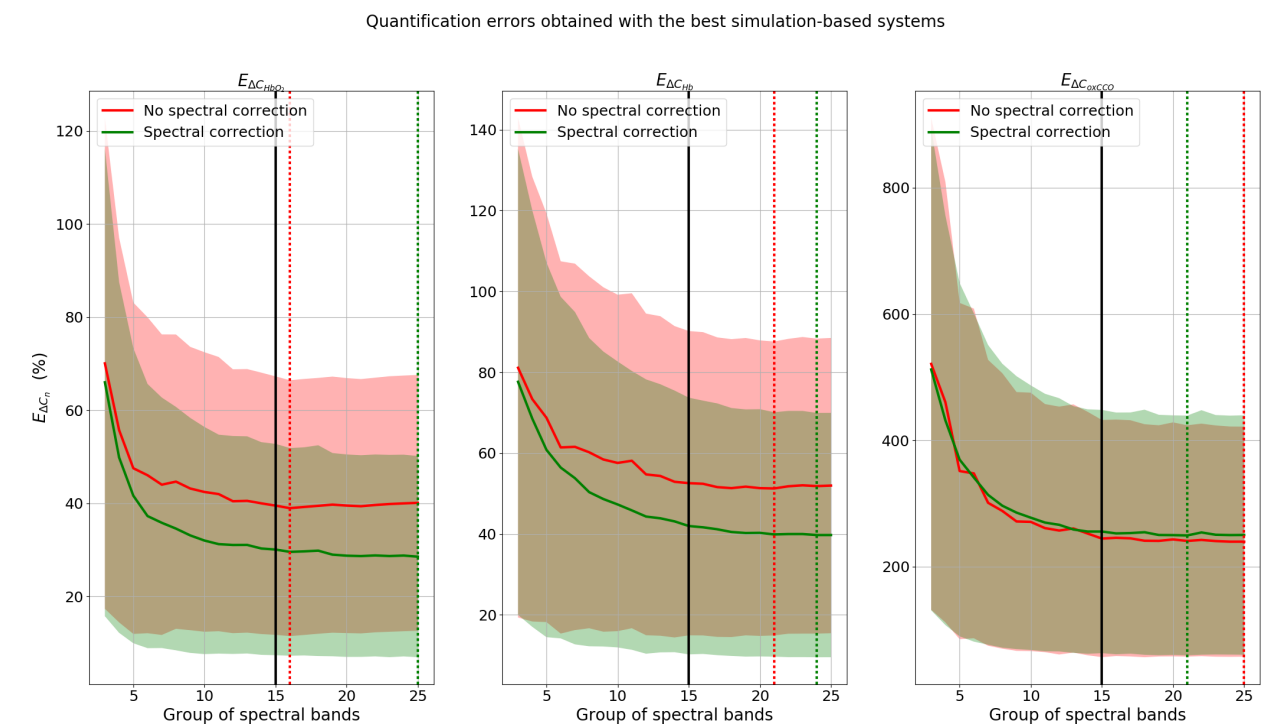

Figure 6. Quantification errors (see Equation (10)) in $\Delta C_{\mathrm{HbO}_{2}}, \Delta \mathrm{C}_{\mathrm{Hb}}$, and $\Delta \mathrm{C}_{\mathrm{oxCCO}}$ obtained for the best simulation-based deconvolution systems of $N$ spectral bands $(N \in[3 ; 25])$. The solid lines stand for the mean quantification error. The colored areas represent the dispersion range of the quantification errors. The vertical dashed lines indicate the optimal number of spectral bands for the deconvolution of each chromophore. Both corrected (in green) and uncorrected spectral configuration (in red) are represented.

For the three chromophores, the quantification errors are important when a small number of spectral bands are used, but the quantification errors decrease with a larger number of bands. The quantification errors in $\Delta C_{\mathrm{HbO}_{2}}$ are on average 30\% lower when the spectral correction is applied than when it is not applied. The quantification errors in $\Delta C_{H b}$ are on average $20 \%$ lower when the spectral correction is applied than when it is not applied. The quantification errors in $\Delta C_{o x C C O}$ are 
equivalent with and without spectral correction. We can notice that the quantification errors reach a plateau when 15 corrected and uncorrected spectral bands are used. These spectral bands are indicated by violet points in Figure 7 and will be named optimal reduced spectral bands in the rest of the paper.
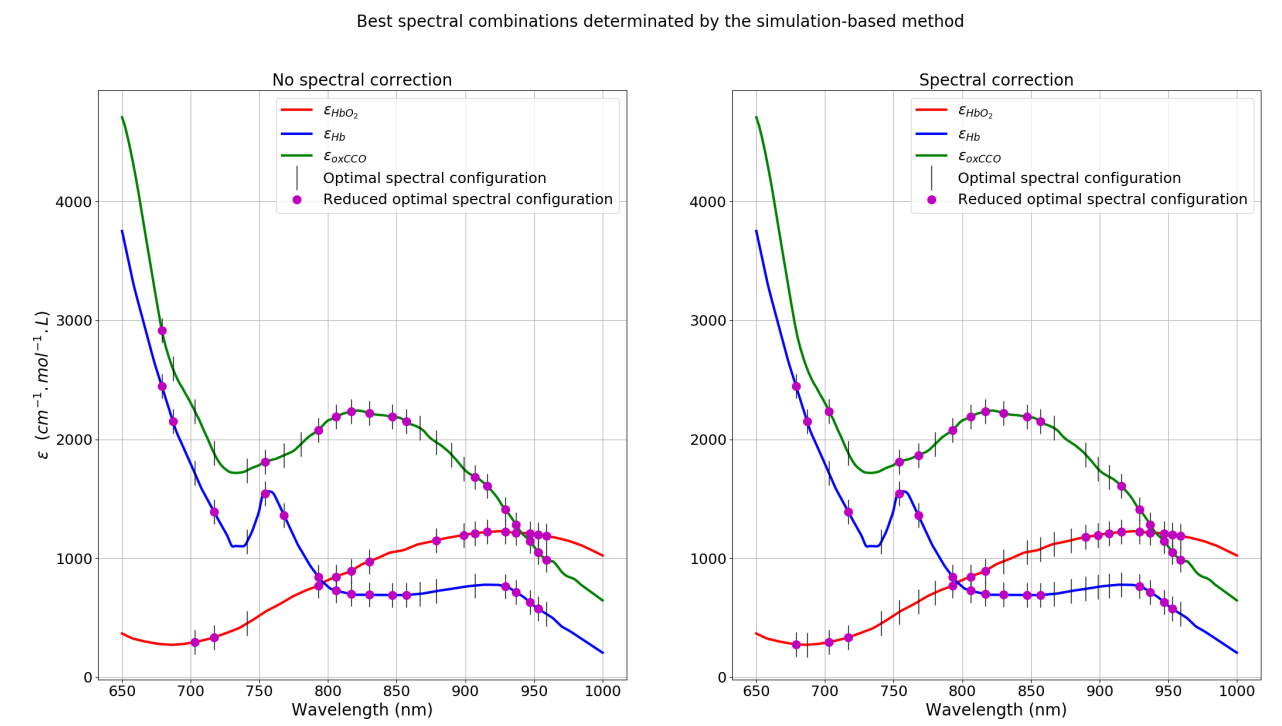

Figure 7. Optimal spectral configuration of the hyperspectral camera XIMEA MQ022HG-IM-SM5X5NIR obtained with the simulation-based method. The peaks of the selected spectral bands are represented as vertical lines in the molar extinction coefficients of the deconvolved chromophores. The reduced optimal spectral bands are indicated by violet points in the molar extinction coefficients of the deconvolved chromophores.

For each chromophore, different optimal spectral combinations are found. For the quantification of $\Delta C_{\mathrm{HbO}_{2}}$, the best spectral configuration of our hyperspectral camera is composed of 25 spectral bands if the spectral correction is applied and of 16 spectral bands if the spectral correction is not applied. These spectral bands are indicated by black vertical lines in the $\mathrm{HbO}_{2}$ molar extinction spectra in Figure 7. For these configurations, the quantification error (with Monte Carlo and intensity noise addition) is equal to $28.7 \% \pm 21.4 \%$ (mean \pm standard deviation of the $10^{3}$ measurements) when the spectral correction is applied and is equal to $38.5 \% \pm 26.9 \%$ when the spectral correction is not applied.

For the quantification of $\Delta C_{H b}$, the best spectral configuration of our hyperspectral camera is composed of 24 spectral bands if the spectral correction is applied and of 21 spectral bands if the spectral correction is not applied. These spectral bands are indicated by black vertical lines in the $\mathrm{Hb}$ molar extinction spectra in Figure 7. For these configurations, the quantification error (with Monte Carlo and intensity noise addition) is equal to $39 \% \pm 29.7 \%$ (mean \pm standard deviation of the $10^{3}$ measurements) when the spectral correction is applied and is equal to $49.7 \% \pm 36.6 \%$ when the spectral correction is not applied.

For the quantification of $\Delta C_{o x C C O}$, the best spectral configuration of our hyperspectral camera is composed of 21 spectral bands if the spectral correction is applied and of 25 spectral bands if the spectral correction is not applied. These spectral bands are indicated by black vertical lines in the oxCCO molar extinction spectra in Figure 7. For these configurations, the quantification error (with Monte Carlo and intensity noise addition) is equal to $251.1 \% \pm 195.4 \%$ (mean \pm standard deviation of the $10^{3}$ measurements) when the spectral correction is applied and is equal to $237.6 \% \pm 183.1 \%$ when the spectral correction is not applied.

\section{Quantification Performances of Deconvolution Systems}

When using the optimal or the reduced optimal spectral configurations of the hyperspectral camera (see Figure 7), three linear systems have to be defined to independently quantify $\Delta C_{\mathrm{HbO}_{2}}$, $\Delta C_{H b}$, and $\Delta C_{o x C C O}$. In the rest of the paper, these systems will be named multiple deconvolution 
systems. When inspecting the spectral band used in the optimal reduced multiple systems, twenty-one different spectral bands are used with and without spectral correction. Thus, a single system can be designed using these spectral bands to quantify $\Delta C_{\mathrm{HbO}_{2}}, \Delta C_{\mathrm{Hb}}$, and $\Delta C_{\mathrm{OxCCO}}$ in a single measurement. In the same way, when inspecting the spectral variables used in the optimal multiple systems, all spectral bands of the hyperspectral camera are used. The accuracy of the quantification of the chromophore $n$ can be measured using the mean and the standard deviation of the quantification error (see Equation (10)). According to ISO 5725-1standards, the term "accuracy" stands for the trueness of the measurement (mean quantification errors close to $0 \%$ ) and the repeatability of the measurement (standard deviation of quantification errors close to $0 \%$ ). The mean and standard deviation values were calculated on $10^{3}$ noisy measurements. These metrics were calculated using the multiple and single deconvolution systems of the hyperspectral camera; see Table 3.

Table 3. Quantification performances of the multiple and single deconvolution systems of the hyperspectral camera.

\begin{tabular}{cccccc}
\hline & & \multicolumn{2}{c}{ Multiple Deconvolution Systems } & \multicolumn{2}{c}{ Single Deconvolution Systems } \\
\hline & & Optimal & Optimal Reduced & Optimal Reduced & All Spectral Bands \\
\hline \multirow{5}{*}{ Spectral correction } & $m\left(E_{\Delta C_{H b O_{2}}}\right)$ & 28.7 & 29.7 & 29.1 & 28.7 \\
& $\sigma\left(E_{\Delta C_{H b O_{2}}}\right)$ & 21.4 & 23.1 & 21.9 & 21.4 \\
\cline { 2 - 6 } & $m\left(E_{\Delta C_{H b}}\right)$ & 39 & 39.7 & 39.4 & 39 \\
& $\sigma\left(E_{\Delta C_{H b}}\right)$ & 29.7 & 31.1 & 29.8 & 29.7 \\
\cline { 2 - 6 } No spectral correction & $m\left(E_{\Delta C_{o x c C O}}\right)$ & 251.1 & 252.1 & 250.1 & 250.1 \\
& $\sigma\left(E_{\Delta C_{o x C C O}}\right)$ & 195.4 & 198.9 & 194 & 195.2 \\
\hline & $m\left(E_{\Delta C_{H b O}}\right)$ & 38.5 & 38.7 & 40.6 & 40.6 \\
& $\sigma\left(E_{\Delta C_{H b O}}\right)$ & 26.9 & 27.8 & 28.1 & 27.3 \\
\cline { 2 - 6 } & $m\left(E_{\Delta C_{H b}}\right)$ & 49.7 & 50.9 & 50.5 & 36.1 \\
& $\sigma\left(E_{\Delta C_{H b}}\right)$ & 36.6 & 37.5 & 36.8 & 237.6 \\
\cline { 2 - 6 } & $m\left(E_{\Delta C_{o x c C O}}\right)$ & 237.6 & 244.3 & 183.9 & 183.1 \\
\hline
\end{tabular}

For each chromophore, the lowest values of the mean and standard deviation of the quantification errors in $\Delta C_{\mathrm{HbO}_{2}}$ and $\Delta C_{\mathrm{Hb}}$ are obtained with the optimal multiple deconvolution system using corrected spectral bands. When the spectral bands are not corrected, the mean and standard deviation values of the quantification errors in $\Delta C_{\mathrm{HbO}_{2}}$ and $\Delta C_{\mathrm{Hb}}$ are higher than the values obtained with corrected spectral bands. However, we can notice that the mean and standard deviation values of the quantification errors in $\Delta C_{o x C C O}$ computed without spectral correction are lower than the values obtained with corrected spectral bands. The quantification errors computed with the optimal multiple system are similar to those obtained with the single system composed of all the spectral bands of the hyperspectral camera. The quantification errors computed with the optimal reduced multiple system are similar to those obtained with the single optimal reduced system.

\subsection{Impact of the Signal-to-Noise Ratio in the Measurements}

As mentioned in Section 2.3, the SNR values of the imaging system directly impact the amount of noise and the accuracy of the simulated quantities. To illustrate the effect of SNR on the measurements, the mean and standard deviation of the quantification errors in $\Delta C_{\mathrm{HbO}_{2}}, \Delta C_{\mathrm{Hb}}$ and $\Delta C_{o x C C O}$ (see Equation (10)) obtained for the optimal spectral configuration of the hyperspectral camera (see Figure 7) and the RGB camera are represented as a function of SNR; see Figures 8 and 9. For each chromophore system, the mean and standard deviation values of the quantification errors are high for low SNR values and decrease with increasing SNR values. 
Mean quantification error estimated for the RGB camera and optimal configurations of the hyperspectral camera as a function of SNR
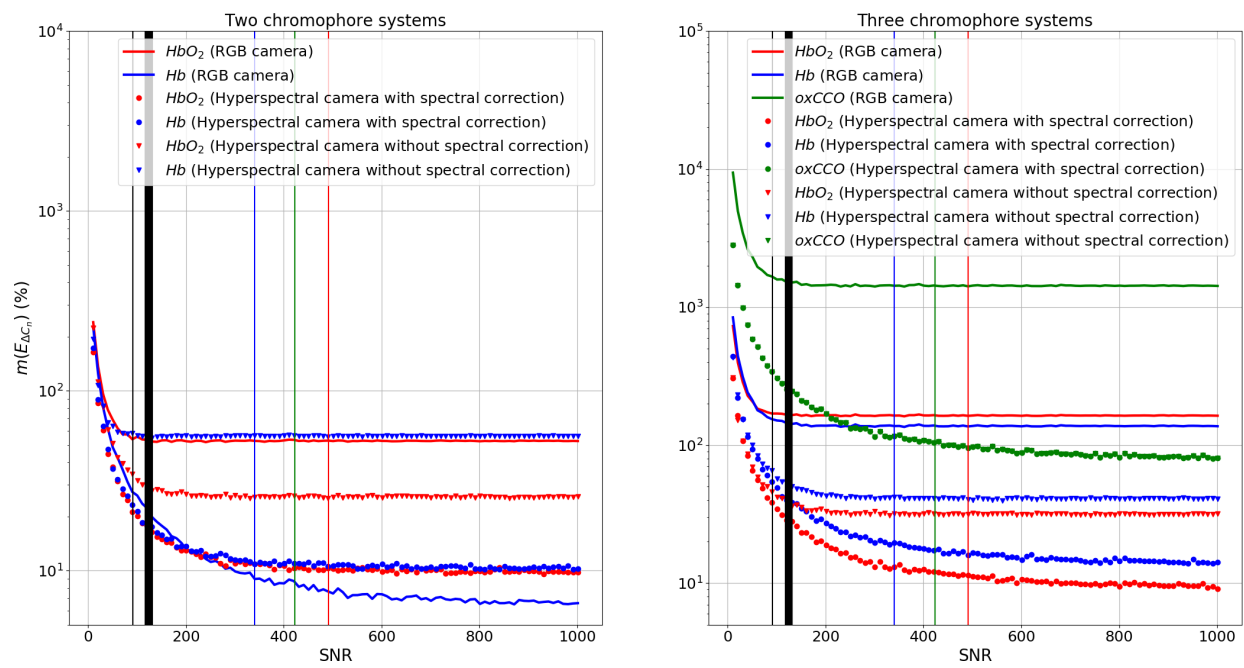

Figure 8. Mean quantification errors in $\Delta C_{\mathrm{HbO}_{2}}$ (b), $\Delta C_{\mathrm{Hb}}$ (blue curves), and $\Delta C_{o x C C O}$ (green curves) (see Equation (10)) obtained for the optimal spectral configuration of the hyperspectral camera (see Figure 7) and the RGB camera as a function of SNR. The solid lines were computed using the RGB camera; the curves with circles and with triangles were computed using the hyperspectral camera with and without spectral correction, respectively. Red, green, and blue vertical lines indicate the SNR values of the R, G, and B channels of the RGB camera and black vertical lines the SNR values of the spectral channels of the hyperspectral camera.
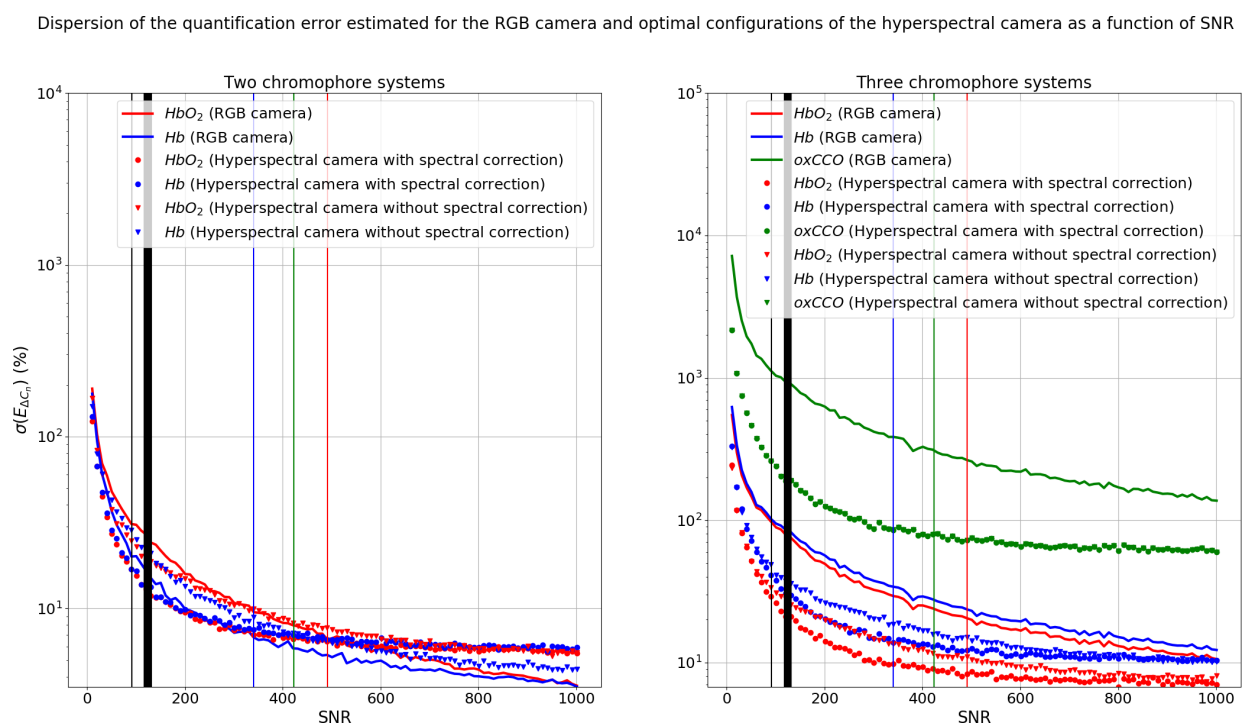

Figure 9. Standard deviation of the quantification errors in $\Delta C_{\mathrm{HbO}_{2}}$ (red curves), $\Delta C_{\mathrm{Hb}}$ (blue curves), and $\Delta C_{o x C C O}$ (green curves) (see Equation (10)) obtained for the optimal spectral configuration of the hyperspectral camera (see Figure 7) and the RGB camera as a function of SNR. The solid lines were computed using the RGB camera; the curves with circles and with triangles were computed using the hyperspectral camera with and without spectral correction, respectively. Red, green, and blue vertical lines indicate the SNR values of the R, G, and B channels of the RGB camera and black vertical lines the SNR values of the spectral channels of the hyperspectral camera.

When a two-chromophore system is considered with the RGB camera (SNR = 10), the quantification errors in $\Delta C_{\mathrm{HbO}_{2}}$ and $\Delta C_{\mathrm{Hb}}$ are equal to $236 \% \pm 187 \%$ and $226 \% \pm 179 \%$, respectively. When the SNR value is equal to 1000, the quantification errors in $\Delta C_{\mathrm{HbO}_{2}}$ and $\Delta C_{\mathrm{Hb}}$ are equal to $52 \% \pm 3 \%$ and $6 \% \pm 3 \%$, respectively. For high SNR values, it is interesting to note that the 
quantification of $\Delta \mathrm{C}_{\mathrm{HbO}_{2}}$ is not accurate (low $\sigma\left(E_{\mathrm{HbO}_{2}}\right)$ values and high $m\left(E_{\mathrm{HbO}_{2}}\right)$ values) and the quantification of $\Delta C_{\mathrm{Hb}}$ is accurate. Moreover, the mean quantification errors in $\Delta C_{\mathrm{HbO}_{2}}$ reach a plateau for an SNR value equal to 110. For the hyperspectral camera with an SNR value equal to 10, the quantification errors in $\Delta C_{\mathrm{HbO}_{2}}$ are equal to $232 \% \pm 173 \%$ when the spectral correction is applied and $259 \% \pm 193 \%$ when it is not applied. The quantification errors in $\Delta C_{H b}$ are equal to $235 \% \pm 176 \%$ when the spectral correction is applied and $267 \% \pm 201 \%$ when it is not applied. When the SNR value is equal to 1000, the quantification errors in $\Delta C_{\mathrm{HbO}_{2}}$ are equal to $8 \% \pm 6 \%$ when the spectral correction is applied and $28 \% \pm 6 \%$ when it is not applied. The quantification errors in $\Delta C_{H b}$ are equal to $8 \% \pm 6 \%$ when the spectral correction is applied and $52 \% \pm 5 \%$ when it is not applied. For high SNR values, the quantification of $\Delta C_{\mathrm{HbO}_{2}}$ and $\Delta \mathrm{C}_{\mathrm{Hb}}$ is accurate when the spectral correction is applied and not accurate when the spectral correction is not applied (low $\sigma\left(E_{n}\right)$ values and high $m\left(E_{n}\right)$ values). When the spectral correction is not applied, the mean quantification errors in $\Delta C_{\mathrm{HbO}_{2}}$ and $\Delta C_{\mathrm{Hb}}$ reach a plateau for an SNR value equal to 200 and 110, respectively.

When a three-chromophore system is considered with the RGB camera (SNR $=10)$, the quantification errors in $\Delta C_{\mathrm{HbO}_{2}}, \Delta C_{\mathrm{Hb}}$, and $\Delta C_{\mathrm{oxCCO}}$ are equal to $712 \% \pm 531 \%, 822 \% \pm 617 \%$, and $9427 \% \pm 7011 \%$, respectively. When the SNR value is equal to 1000, the quantification errors in $\Delta C_{\mathrm{HbO}_{2}}, \Delta C_{\mathrm{Hb}}$, and $\Delta C_{o x C C O}$ are equal to $164 \% \pm 10 \%, 138 \% \pm 12 \%$, and $1431 \% \pm 138 \%$, respectively. The mean quantification errors in $\Delta C_{\mathrm{HbO}_{2}}, \Delta C_{\mathrm{Hb}}$, and $\Delta C_{o x C C O}$ reach a plateau for an SNR value equal to 110, 130, and 130, respectively. For high SNR values, the quantification of $\Delta C_{\mathrm{HbO}_{2}}, \Delta C_{\mathrm{Hb}}$, and $\Delta C_{o x C C O}$ is not accurate (low $\sigma\left(E_{n}\right)$ values and high $m\left(E_{n}\right)$ values). For the hyperspectral camera with an SNR value equal to 10, the quantification errors in $\Delta C_{\mathrm{HbO}_{2}}$ are equal to $345 \% \pm 267 \%$ when the spectral correction is applied and $328 \% \pm 248 \%$ when it is not applied. The quantification errors in $\Delta C_{H b}$ are equal to $486 \% \pm 379 \%$ when the spectral correction is applied and $496 \% \pm 382 \%$ when it is not applied. The quantification errors in $\Delta C_{o x C C O}$ are equal to $2908 \% \pm 2190 \%$ when the spectral correction is applied and $2951 \% \pm 2201 \%$ when it is not applied. When the SNR value is equal to 1000 , the quantification errors in $\Delta \mathrm{C}_{\mathrm{HbO}_{2}}$ are equal to $10 \% \pm 8 \%$ when the spectral correction is applied and $33 \% \pm 8 \%$ when it is not applied. The quantification errors in $\Delta C_{H b}$ are equal to $14 \% \pm 11 \%$ when the spectral correction is applied and $41 \% \pm 11 \%$ when it is not applied. The quantification errors in $\Delta C_{\text {oxCCO }}$ are equal to $90 \% \pm 68 \%$ when the spectral correction is applied and $91 \% \pm 70 \%$ when it is not applied. For high SNR values, the quantification of $\Delta C_{\mathrm{HbO}_{2}}$ and $\Delta C_{\mathrm{Hb}}$ is accurate when the spectral correction is applied and not accurate (low $\sigma\left(E_{n}\right)$ values and high $m\left(E_{n}\right)$ values) when the spectral correction is not applied. The quantification of $\Delta C_{o x C C O}$ is not accurate with and without spectral correction (low $\sigma\left(E_{o x C C O}\right)$ values and high $m\left(E_{o x C C O}\right)$ values). We can notice that the measurement uncertainty of $\triangle C_{o x C C O}$ is similar for all SNR values with and without spectral correction. When the spectral correction is not applied, the mean quantification errors in $\Delta C_{\mathrm{HbO}_{2}}$ and $\Delta C_{\mathrm{Hb}}$ reach a plateau for an SNR value equal to 200 .

\subsection{Hemodynamic Monitoring}

The hemodynamic monitoring $\left(\Delta \mathrm{C}_{\mathrm{HbO}_{2}}\right.$ and $\Delta \mathrm{C}_{\mathrm{Hb}}$ measurements) following a simulated patient neuronal stimulation (see Section 2.7) is represented In Figures 10 and 11. In Figure 10, the $\Delta C_{\mathrm{HbO}_{2}}$ and $\Delta C_{H b}$ values were computed with the optimal spectral combination of the hyperspectral camera; see Figure 7. In Figure 11, the $\Delta C_{\mathrm{HbO}_{2}}$ and $\Delta C_{\mathrm{Hb}}$ values were computed with the RGB camera. In these figures, the modified Beer-Lambert law was computed $10^{3}$ times, using different Monte Carlo and intensity noise occurrences for each time iteration. When using the 121 wavelengths included between $780 \mathrm{~nm}$ and $900 \mathrm{~nm}$ [23] to quantify the concentration changes during the stimulation period (from $t=18 \mathrm{~s}$ to $t=38 \mathrm{~s}$ ), there is a $12 \%$ overestimation of the $\Delta C_{\mathrm{HbO}_{2}}$ values and a $34 \%$ underestimation of the $\Delta C_{H b}$ values compared to the theoretical measurements. When using the eight wavelengths identified by Arifler et al. [27] to quantify the concentration changes during the stimulation period, there is a $13 \%$ overestimation of the $\Delta C_{\mathrm{HbO}_{2}}$ values and a $31 \%$ underestimation of the $\Delta C_{\mathrm{Hb}}$ values compared to the theoretical measurements. 

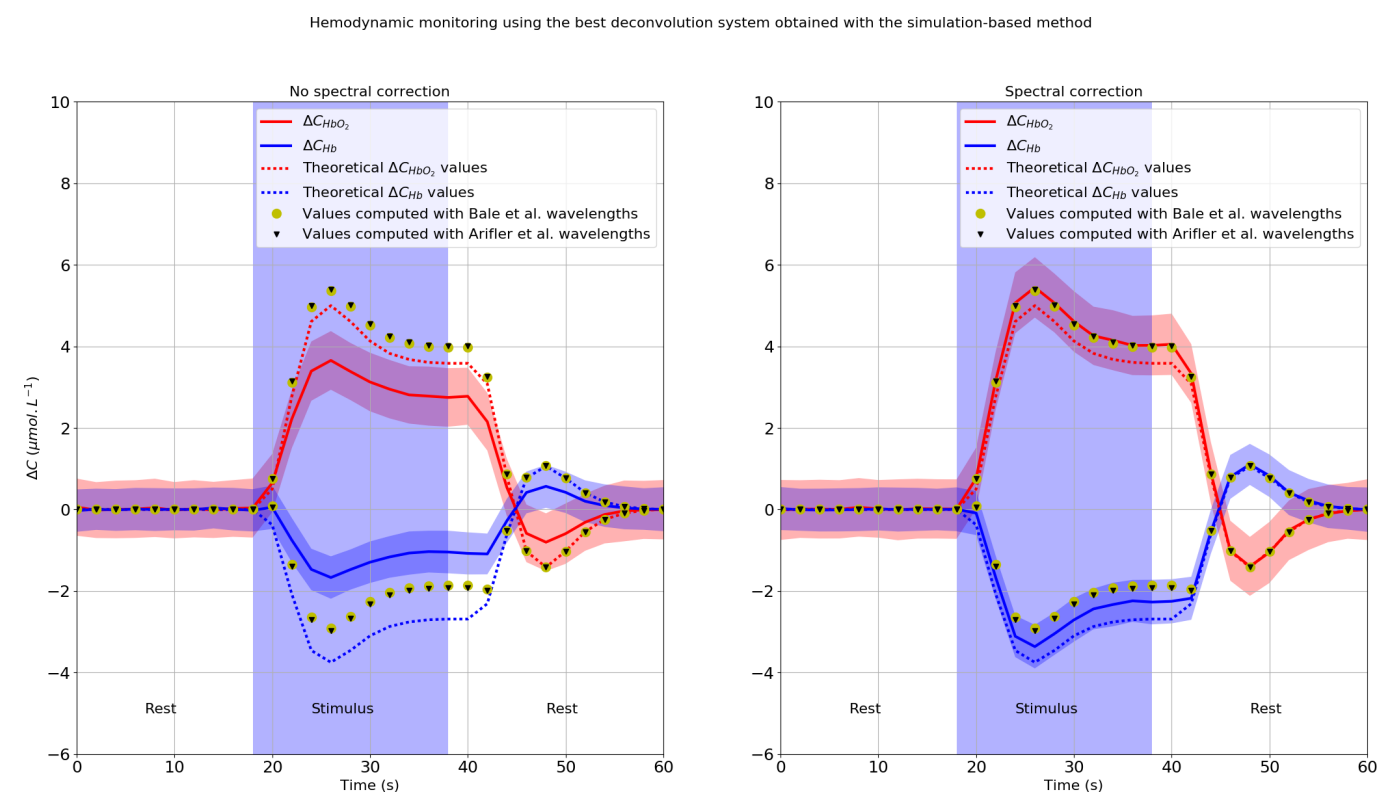

Figure 10. Hemodynamic monitoring following neuronal activation computed with the spectral configuration of the hyperspectral camera obtained with the simulation-based method (see Figure 7). The dashed lines represent the theoretical hemodynamic responses following the neuronal stimulation. The dispersion ranges of the measurements are represented by colored areas. The concentration changes averaged over the $10^{3}$ noisy measurements are represented in solid lines. The concentration changes' time courses computed with the 121 wavelengths included between $780 \mathrm{~nm}$ and $900 \mathrm{~nm}$ [23] are represented with yellow points. The ones computed with Arifler et al.'s wavelengths [27] are represented with black triangles. The blue rectangle represents the "patient" physiological stimulus.

In Figure 10, the hemodynamic monitoring following a simulated neuronal stimulation was computed using hyperspectral imaging. The optimal corrected and uncorrected spectral configurations of the hyperspectral camera were used to quantify the $\Delta \mathrm{C}_{\mathrm{HbO}_{2}}$ and $\Delta \mathrm{C}_{\mathrm{Hb}}$ values. The quantification dispersion ranges of the corrected and uncorrected spectral configurations have approximately the same range of magnitude. The standard deviation averaged over all time measurements for $\Delta \mathrm{C}_{\mathrm{HbO}_{2}}$ and $\Delta C_{H b}$ are equal to $0.9 \mu \mathrm{mol} \cdot \mathrm{L}^{-1}$ and $0.7 \mu \mathrm{mol} \cdot \mathrm{L}^{-1}$, respectively. When the spectral bands are corrected, the $\Delta \mathrm{C}_{\mathrm{HbO}_{2}}$ and $\Delta \mathrm{C}_{\mathrm{Hb}}$ values averaged over the $10^{3}$ noisy measurements have a good match with the theoretical hemodynamic responses. During the stimulation period, there is on average a $14 \%$ overestimation of the $\Delta C_{\mathrm{HbO}_{2}}$ values and a $18 \%$ underestimation of the $\Delta C_{\mathrm{Hb}}$ values compared to the theoretical measurements. When the spectral bands are not corrected, there is on average a $17 \%$ underestimation of the $\Delta C_{\mathrm{HbO}_{2}}$ values and a $58 \%$ underestimation of the $\Delta C_{H b}$ values compared to the theoretical measurements. The correlation coefficients computed between the theoretical $\mathrm{HbO}_{2}$ response and the $\Delta \mathrm{C}_{\mathrm{HbO}_{2}}$ time courses are higher when the spectral bands are corrected $(r=0.951 \pm 0.013)$ than when they are not $(r=0.904 \pm 0.026)$. In the same way, the correlation coefficients computed between the theoretical $\mathrm{Hb}$ response and the $\Delta C_{H b}$ time courses are higher when the spectral bands are corrected $(r=0.932 \pm 0.018)$ than when they are not $(r=0.783 \pm 0.059)$. The correlation coefficient values are summarized in Table 4.

In Figure 11, the hemodynamic monitoring following a simulated neuronal stimulation was computed using RGB imaging. The standard deviation averaged over all time measurements is equal to $0.35 \mu \mathrm{mol} \cdot \mathrm{L}^{-1}$ and $0.20 \mu \mathrm{mol} \cdot \mathrm{L}^{-1}$ for $\Delta C_{\mathrm{HbO}_{2}}$ and $\Delta C_{\mathrm{Hb}}$, respectively. The $\Delta C_{\mathrm{HbO}_{2}}$ values values averaged over the $10^{3}$ noisy measurements do not have a good match with the theoretical $\mathrm{HbO}_{2}$ response; however, the match is rather good between the $\Delta C_{\mathrm{Hb}}$ values and the theoretical $\mathrm{Hb}$ response. During the stimulation period, there is on average a $48 \%$ underestimation of the $\Delta C_{\mathrm{HbO}_{2}}$ values and a $4 \%$ underestimation of the $\Delta C_{H b}$ values compared to the theoretical measurements. The correlation coefficients computed between the theoretical $\mathrm{HbO}_{2}$ response and the $\Delta \mathrm{C}_{\mathrm{HbO}_{2}}$ time courses are equal to 
$r=0.949 \pm 0.013$. The correlation coefficients computed between the theoretical $\mathrm{Hb}$ response and the $\Delta C_{H b}$ time courses are equal to $r=0.989 \pm 0.002$. The correlation coefficient values are summarized in Table 4.

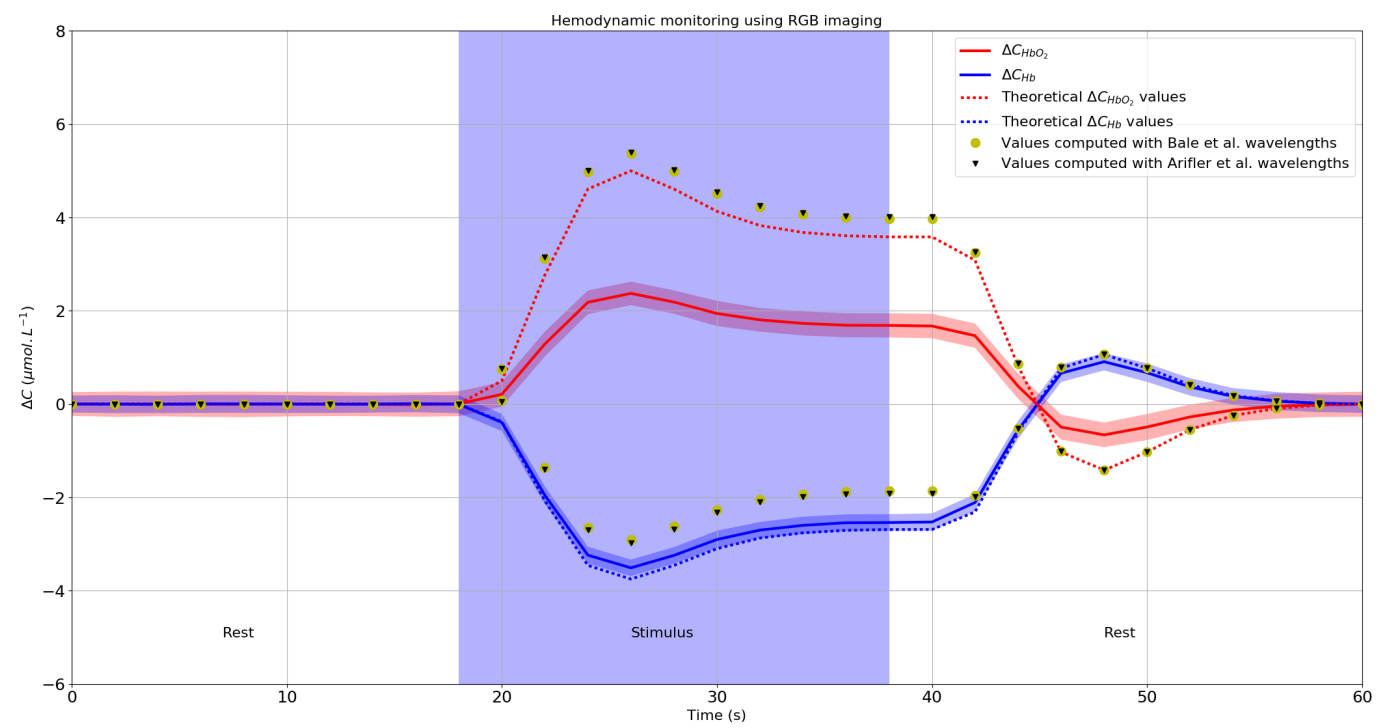

Figure 11. Hemodynamic monitoring following neuronal activation computed with RGB imaging. The dashed lines represent the theoretical hemodynamic responses following the neuronal stimulation. The dispersion ranges of the measurements are represented by colored areas. The concentration changes averaged over the $10^{3}$ noisy measurements are represented in solid lines. The concentration changes' time courses computed with the 121 wavelengths included between $780 \mathrm{~nm}$ and $900 \mathrm{~nm}$ [23] are represented with yellow points. The ones computed with Arifler et al.'s wavelengths [27] are represented with black triangles. The blue rectangle represents the "patient" physiological stimulus.

Table 4. Pearson correlation coefficients computed between the theoretical hemodynamic responses and the simulated concentration changes' time courses; see Figures 10 and $11 . \mu\left(r_{n}\right)$ and $\sigma\left(r_{n}\right)$ designate the mean and standard deviation, respectively, of the Pearson correlation coefficients computed with the $10^{3}$ noisy time courses of the chromophore $n$.

\begin{tabular}{cccc}
\hline & \multirow{2}{*}{ RGB Imaging } & \multicolumn{2}{c}{ Hyperspectral Imaging } \\
\cline { 3 - 4 } & & Spectral Correction & No Spectral Correction \\
\hline$\mu\left(r_{\mathrm{HbO}_{2}}\right)$ & 0.949 & 0.951 & 0.904 \\
$\sigma\left(r_{\mathrm{HbO}_{2}}\right)$ & 0.013 & 0.013 & 0.026 \\
\hline$\mu\left(r_{\mathrm{Hb}}\right)$ & 0.989 & 0.932 & 0.783 \\
$\sigma\left(r_{\mathrm{Hb}}\right)$ & 0.002 & 0.018 & 0.059 \\
\hline
\end{tabular}

\subsection{Hemodynamic and Metabolic Monitoring}

The hemodynamic and metabolic monitoring $\left(\Delta C_{\mathrm{HbO}_{2}}, \Delta \mathrm{C}_{\mathrm{Hb}}\right.$, and $\Delta \mathrm{C}_{\mathrm{oxCCO}}$ measurements) following a simulated patient neuronal stimulation (see Section 2.7) are represented in Figures 12 and 13. In Figure 12, the $\Delta C_{\mathrm{HbO}_{2}}, \Delta C_{\mathrm{Hb}}$, and $\Delta C_{o x C C O}$ values were computed with the optimal spectral combination of the hyperspectral camera; see Figure 7. In Figure 13, the $\Delta C_{\mathrm{HbO}_{2}}, \Delta C_{\mathrm{Hb}}$, and $\Delta C_{o x C C O}$ values were computed with the RGB camera. In these figures, the modified Beer-Lambert law was computed $10^{3}$ times, using different Monte Carlo and intensity noise occurrences for each time iteration. When using the 121 wavelengths included between $780 \mathrm{~nm}$ and $900 \mathrm{~nm}$ [23] to quantify the concentration changes during the stimulation period (from $t=18 \mathrm{~s}$ to $t=38 \mathrm{~s}$ ), there is a $1.2 \%$ underestimation of the $\Delta C_{\mathrm{HbO}_{2}}$ values, a $0.4 \%$ overestimation of the $\Delta C_{\mathrm{Hb}}$ values, and a $1.3 \%$ overestimation of the $\Delta C_{o x C C O}$ values compared to the theoretical measurements. 
When using the eight wavelengths identified by Arifler et al. [27] to quantify the concentration changes during the stimulation period, there is a $1.7 \%$ underestimation of the $\Delta C_{\mathrm{HbO}_{2}}$ values, a $1.91 \%$ overestimation of the $\Delta C_{H b}$ values, and a $6 \%$ overestimation of the $\Delta C_{o x C C O}$ values compared to the theoretical measurements.

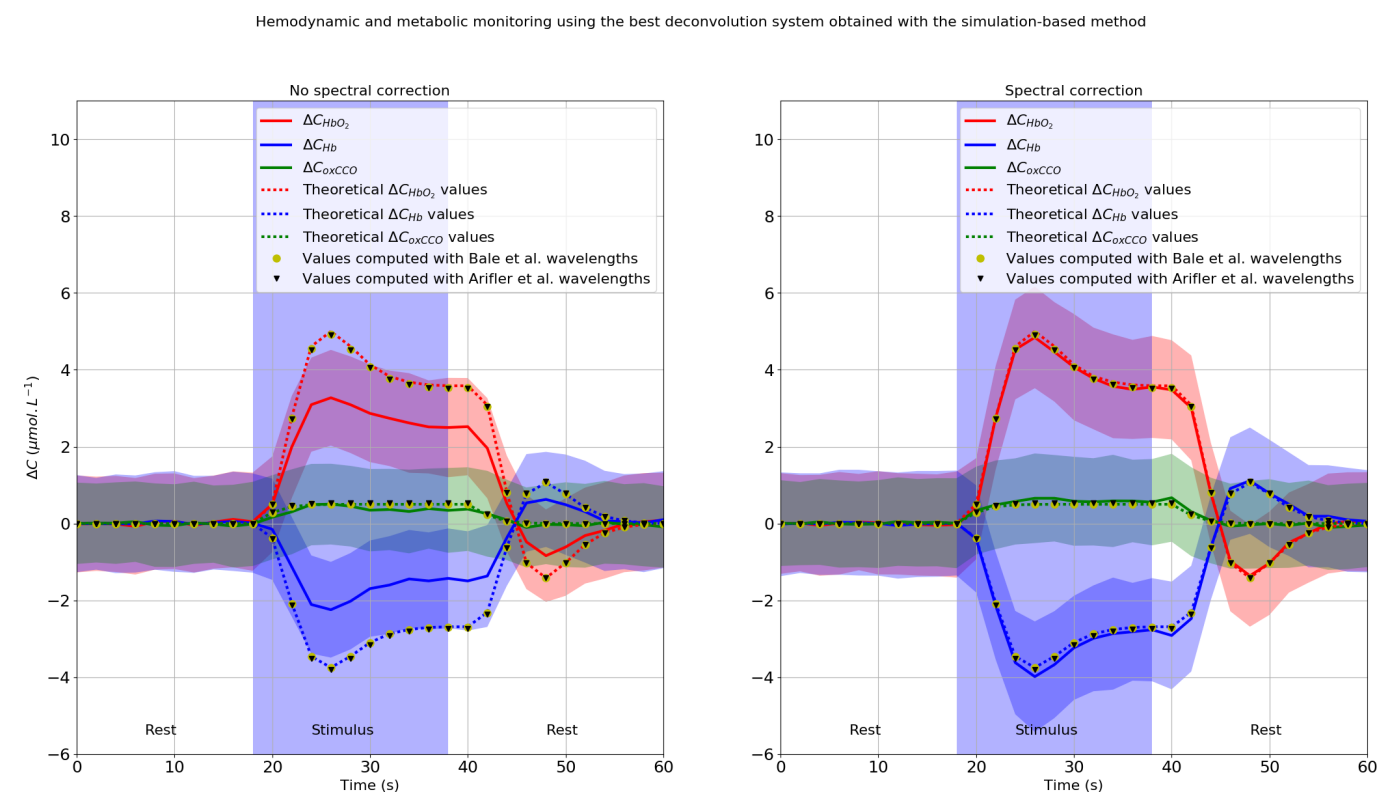

Figure 12. Hemodynamic and metabolic monitoring following neuronal activation computed with the optimal spectral configuration obtained with the simulation-based method (see Figure 7). The dashed lines represent the theoretical hemodynamic and metabolic responses following the neuronal stimulation. The dispersion ranges of the measurements are represented by colored areas. The concentration changes averaged over the $10^{3}$ noisy measurements are represented in solid lines. The concentration changes' time courses computed with the 121 wavelengths included between $780 \mathrm{~nm}$ and $900 \mathrm{~nm}$ [23] are represented with yellow points. The ones computed with Arifler et al.'s wavelengths [27] are represented with black triangles. The blue rectangle represents the "patient" physiological stimulus.

In Figure 12, the hemodynamic and metabolic monitoring following a simulated neuronal stimulation was computed using hyperspectral imaging. The optimal corrected and uncorrected spectral configurations of the hyperspectral camera were used to quantify the $\Delta C_{\mathrm{HbO}_{2}}, \Delta C_{\mathrm{Hb}}$, and $\Delta C_{o x C C O}$ values. The quantification dispersion ranges of the corrected and uncorrected spectral configurations have approximately the same range of magnitude. The standard deviation averaged over all time measurements for $\Delta C_{\mathrm{HbO}_{2}}, \Delta C_{\mathrm{Hb}}$, and $\Delta C_{o x \mathrm{CCO}}$ are equal to $1.23 \mu \mathrm{mol} \cdot \mathrm{L}^{-1}, 1.27 \mu \mathrm{mol} \cdot \mathrm{L}^{-1}$, and $1.07 \mu \mathrm{mol} \cdot \mathrm{L}^{-1}$, respectively. When the spectral bands are corrected, the $\Delta \mathrm{C}_{\mathrm{HbO}_{2}}$ and $\Delta \mathrm{C}_{\mathrm{Hb}}$ values averaged over the $10^{3}$ noisy measurements have a good match with the theoretical hemodynamic responses. The $\Delta C_{o x C C O}$ values averaged over the $10^{3}$ noisy measurements have a good match with the theoretical metabolic response with and without spectral correction. When the spectral bands are corrected, there is on average a $0.5 \%$ underestimation of the $\Delta \mathrm{C}_{\mathrm{HbO}_{2}}$ values, a $4.4 \%$ overestimation of the $\Delta C_{H b}$ values, and a $15 \%$ overestimation of the $\Delta C_{o x C C O}$ values compared to the theoretical measurements. When the spectral bands are not corrected, there is on average a $25 \%$ underestimation of the $\Delta C_{\mathrm{HbO}_{2}}$ values, a $42 \%$ underestimation of the $\Delta C_{\mathrm{Hb}}$ values, and a $19 \%$ underestimation of the $\Delta C_{o x C C O}$ values compared to the theoretical measurements. The correlation coefficients computed between the theoretical $\mathrm{HbO}_{2}$ response and the $\Delta \mathrm{C}_{\mathrm{HbO}_{2}}$ time courses are higher when the spectral bands are corrected $(r=0.843 \pm 0.043)$ than when they are not $(r=0.749 \pm 0.068)$. The correlation coefficients computed between the theoretical $\mathrm{Hb}$ response and the $\Delta C_{\mathrm{Hb}}$ time courses are higher when the spectral bands are corrected $(r=0.780 \pm 0.063)$ than when they are not $(r=0.577 \pm 0.112)$. The correlation coefficients computed between the theoretical oxCCO response and the $\Delta C_{o x C C O}$ time 
courses are higher when the spectral bands are corrected $(r=0.256 \pm 0.177)$ than when they are not $(r=0.177 \pm 0.175)$. The correlation coefficient values are summarized in Table 5 .

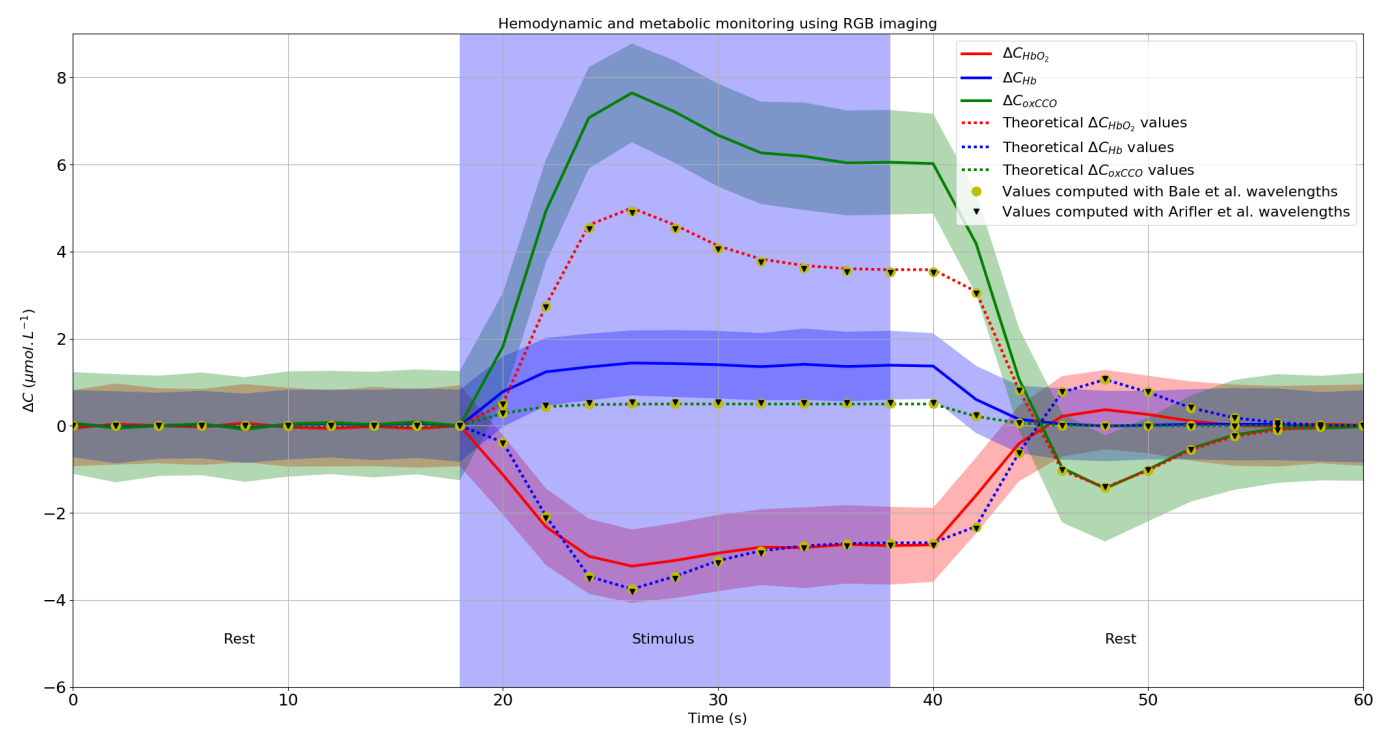

Figure 13. Hemodynamic and metabolic monitoring following neuronal activation computed with RGB imaging. The dashed lines represent the theoretical hemodynamic and metabolic responses following the neuronal stimulation. The dispersion ranges of the measurements are represented by colored areas. The concentration changes averaged over the $10^{3}$ noisy measurements are represented in solid lines. The concentration changes' time courses computed with the 121 wavelengths included between $780 \mathrm{~nm}$ and $900 \mathrm{~nm}$ [23] are represented with yellow points. The ones computed with Arifler et al.'s wavelengths [27] are represented with black triangles. The blue rectangle represents the "patient" physiological stimulus.

In Figure 13, the hemodynamic and metabolic monitoring following a simulated neuronal stimulation was computed using RGB imaging. The standard deviation averaged over all time measurements is equal to $1.24 \mu \mathrm{mol} \cdot \mathrm{L}^{-1}, 1.04 \mu \mathrm{mol} \cdot \mathrm{L}^{-1}$, and $1.54 \mu \mathrm{mol} \cdot \mathrm{L}^{-1}$ for $\Delta C_{\mathrm{HbO}_{2}}, \Delta C_{\mathrm{Hb}}$, and $\Delta C_{o x C C O}$, respectively. The computed $\Delta C_{\mathrm{HbO}_{2}}, \Delta C_{\mathrm{Hb}}$, and $\Delta C_{o x C C O}$ values do not have a good match with the theoretical hemodynamic and metabolic responses. During the stimulation period, there is on average a $169 \%$ underestimation of the $\Delta C_{\mathrm{HbO}_{2}}$ values, a $147 \%$ underestimation of the $\Delta C_{H b}$ values, and a $1036 \%$ overestimation of the $\Delta C_{o x C C O}$ values compared to the theoretical measurements. We can notice that there is an important crosstalk between the chromophores. The $\Delta \mathrm{C}_{\mathrm{HbO}_{2}}$ values were mainly interpreted as $\mathrm{Hb}$ variations, the $\Delta C_{\mathrm{Hb}}$ values as oxCCO variations, and the $\Delta C_{o x C C O}$ as $\mathrm{HbO}_{2}$ variations.

Table 5. Pearson correlation coefficients computed between the theoretical hemodynamic and metabolic responses and the simulated concentration changes' time courses; see Figures 12 and 13. $\mu\left(r_{n}\right)$ and $\sigma\left(r_{n}\right)$ designate the mean and standard deviation, respectively, of the Pearson correlation coefficients computed with the $10^{3}$ noisy time courses of the chromophore $n$.

\begin{tabular}{cccc}
\hline & \multirow{2}{*}{ RGB Imaging } & \multicolumn{2}{c}{ Hyperspectral Imaging } \\
\cline { 3 - 4 } & & Spectral Correction & No Spectral Correction \\
\hline$\mu\left(r_{\mathrm{HbO}_{2}}\right)$ & -0.727 & 0.843 & 0.749 \\
$\sigma\left(r_{\mathrm{HbO}_{2}}\right)$ & 0.072 & 0.043 & 0.068 \\
\hline$\mu\left(r_{\mathrm{Hb}}\right)$ & -0.499 & 0.780 & 0.577 \\
$\sigma\left(r_{\mathrm{Hb}}\right)$ & 0.126 & 0.063 & 0.112 \\
\hline$\mu\left(r_{\text {oxCCO }}\right)$ & 0.880 & 0.256 & 0.177 \\
$\sigma\left(r_{\text {ox CCO }}\right)$ & 0.030 & 0.177 & 0.175 \\
\hline
\end{tabular}




\section{Discussion}

Matcher et al. [46] showed that the performance of spectroscopic analysis can be improved by increasing the number of wavelengths of illumination. The results of our study are consistent with Matcher et al.'s results; see Figure 6. The simulation-based method presented in this paper aimed to identify the optimal spectral combination of our hyperspectral camera for the brain hemodynamic and metabolic monitoring. When the spectral bands are corrected, the optimal spectral configuration is composed of 21 and 22 spectral bands; see Figure 7. This configuration could be however reduced from 10 to 12 spectral bands while keeping fairly constant performances. We can observe a plateau in the quantification errors of $\Delta \mathrm{C}_{\mathrm{HbO}_{2}}$ when 10 or more spectral bands are used. We also can observe a plateau in the quantification errors in $\Delta C_{H b}$ and $\Delta C_{o x C C O}$ when 12 or more spectral bands are used; see Figure 6. This reduction of the number of the spectral bands could be interesting for the real-time computation of hemodynamic and metabolic brain maps in the operative room using commercial hyperspectral cameras and for the conception of dedicated cameras used in functional brain mapping studies.

In this study, the optimal spectral configurations were obtained by searching the three chromophores deconvolution systems that minimize the quantification errors in $\Delta C_{\mathrm{HbO}_{2}}, \Delta C_{\mathrm{Hb}}$, and $\Delta C_{o x C C O}$. Therefore, three different spectral configurations were obtained for the deconvolution of the three chromophores. These spectral configurations, as well as the reference configuration identified by Bale et al. [23] and Arifler et al [27] are represented in Figure 14.

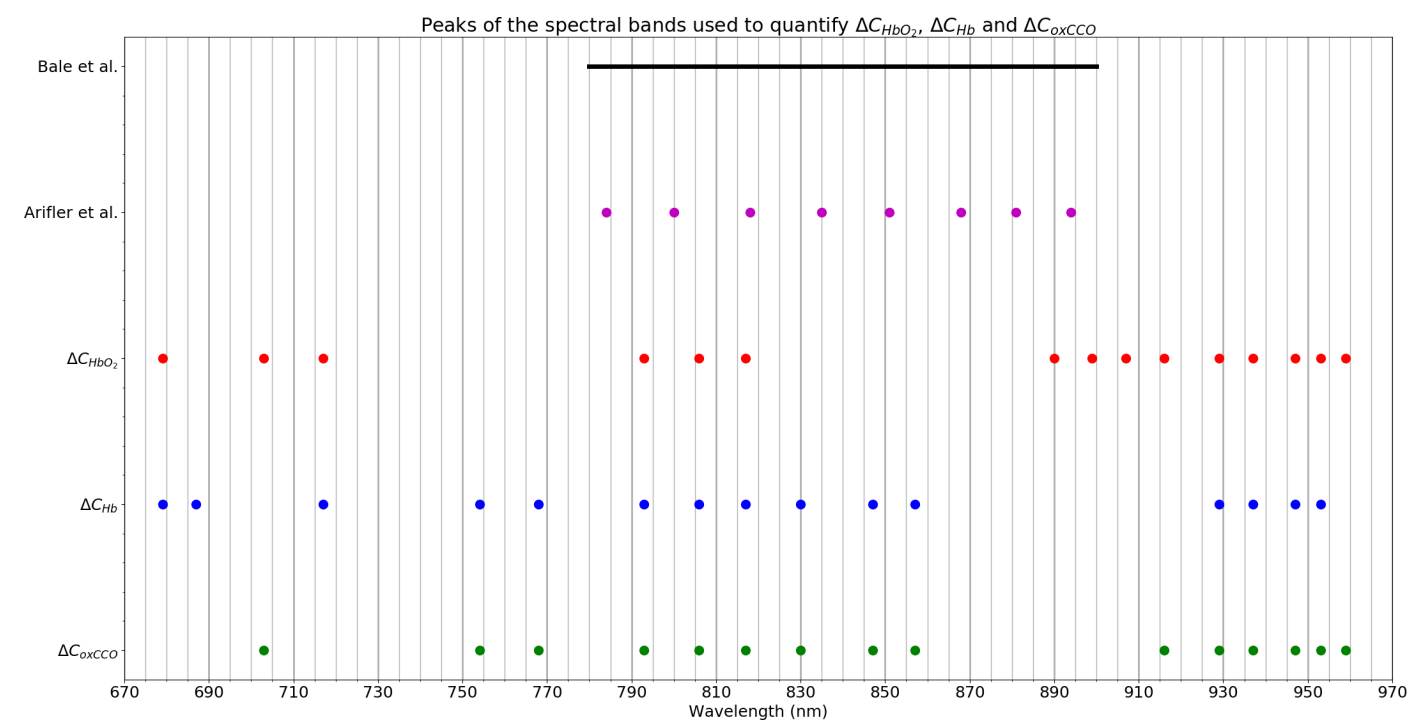

Figure 14. Peaks of spectral bands that minimize the quantification errors in $\Delta C_{\mathrm{HbO}_{2}}, \Delta \mathrm{C}_{\mathrm{Hb}}$ and $\Delta C_{o x C C O}$. The spectral configuration identified by Bale et al. [23] and Arifler et al. [27] are also indicated.

The comparison between the spectral configuration identified by Arifler and al. and ours is not trivial since our hyperspectral camera does not acquire exactly the same wavelengths; see Figure 2. There are some similarities between the wavelengths used in our system and in the system of Arifler et al. Indeed, between $780 \mathrm{~nm}$ and $900 \mathrm{~nm}$, some wavelengths correspond. Our system also used others wavelengths inferior to $780 \mathrm{~nm}(703,754$, and $767 \mathrm{~nm})$ and superior to $900 \mathrm{~nm}(916,929,937$, $947,953$, and $958 \mathrm{~nm})$. The quantification of $\Delta C_{\mathrm{HbO}_{2}}, \Delta C_{\mathrm{Hb}}$, and $\Delta C_{\mathrm{oxCCO}}$ computed with the optimal spectral bands of our hyperspectral camera are consistent with those computed with 121 wavelengths included between $780 \mathrm{~nm}$ and $900 \mathrm{~nm}$ [23] and with those computed with the eight wavelengths identified by Arifler et al. [27]. For a two-chromophore deconvolution system, the quantification error in $\Delta C_{\mathrm{HbO}_{2}}$ measured with our system is equivalent to those measured with 121 wavelengths. However, the choice of our spectral bands aims to reduce the quantification error in $\Delta C_{H b}$; see Figure 10. For a three-chromophore deconvolution system, the quantification errors measured with our system are a little higher than those measured with 121 wavelengths or with Arifler et al.'s wavelengths; see 
Figure 12. This difference may be explained because the illumination and the acquisition of the 121 wavelengths of reference, as well as the eight wavelengths identified by Arifler et al. were simulated as ideal sources and detectors. This means that for these simulations, the term $D \times S$ in Equation (7) is equal to one. To efficiently compare our spectral configuration with these spectral configurations of reference, the spectral sensitivities of the detectors and the illumination sources have to be considered. However, the acquisition condition is different since we acquired the intrinsic signal of an exposed cortex, whereas the wavelengths identified in Bale et al.'s and Arfiler et al.'s studies were identified for functional near-infrared spectroscopy devices.

We showed that a spectral correction (see Section 2.2) is required when a hyperspectral mosaic sensor is used [10]. The spectral correction aims to reduce the quantification errors in the measurements and allows a better follows-up of the temporal hemodynamic and metabolic variations; see Table 5.

We also compared hyperspectral imaging to RGB imaging for the computation of hemodynamic and metabolic brain maps. Hyperspectral imaging is the suitable solution to compute hemodynamic maps and metabolic maps thanks to its ability to acquire the intrinsic signal in the near-infrared range [23]. A very important crosstalk between $\mathrm{HbO}_{2}, \mathrm{Hb}$, and oxCCO can be observed when the RGB camera is used for the computation of hemodynamic and metabolic brain maps; see Figure 13. Therefore, RGB imaging is not a suitable solution to compute metabolic brain maps. An RGB camera could be however a low-cost solution to compute hemodynamic maps; see Figure 11. This solution is not accurate to quantify $\Delta C_{\mathrm{HbO}_{2}}$, but is very accurate for the quantification of $\Delta C_{\mathrm{Hb}}$. This original result is interesting because most surgical microscopes used in the operating room are equipped with standard RGB cameras. It is known that the BOLD signal used in fMRI studies is predominately due to the paramagnetic properties of deoxygenated hemoglobin [47]. This result indicates that the $\Delta C_{H b}$ quantified with RGB imaging can be used in a robust way for intraoperative functional mapping based on SPM analyses.

We incorporated the camera noises in our simulation to find the most robust and reliable spectral configuration. In Figures 8 and 9, we show that the SNR of the imaging system has a drastic impact on quantification performances. An accurate quantification could be obtained with a high SNR value. Therefore, the light source and the camera specifications and settings have to be carefully chosen in order to guarantee an optimal SNR. This simulation framework could be a great tool for industry or researchers working on intraoperative functional brain mapping solutions to help them in the choice of commercial camera. However, our simulation framework needs to be improved. For the moment, a homogeneous volume of grey matter was considered. A realistic mapping of the exposed cortex could be simulated as suggested by Giannoni et al. [28]. This will be considered in future studies. Moreover, the hemodynamic and metabolic changes following neuronal activation were homogeneously simulated in the volume of grey matter. These events are obviously not consistent with those appearing in a real cortical tissue. This modeling also has to be taken into account to improve our method of identification of the optimal spectral bands of a hyperspectral camera for brain hemodynamic and metabolic monitoring.

\section{Conclusions}

We present in this paper a method for the identification of the optimal spectral bands of a commercial camera for the intraoperative monitoring of the brain hemodynamic and metabolic responses following neuronal activation. The method described in the report is based on Monte Carlo simulations of the light propagation in a volume of grey matter and incorporates a realistic modeling of the camera acquisition with the addition of Gaussian noises (experimentally measured with the cameras). We identified that an optimal spectral combination of our hyperspectral camera composed of 21 to 22 spectral bands can be used to compute accurate hemodynamic and metabolic brain maps. This configuration could be however reduced from 10 to 12 spectral bands while keeping fairly constant performances, which is consistent with the spectral configurations proposed in the literature. We also showed that RGB imaging is not a suitable technique to compute metabolic brain 
maps, but is very accurate to compute hemodynamic maps with the quantification of deoxygenated hemoglobin concentration changes. Our Monte Carlo framework needs to be improved, namely with the consideration of the perfusion of grey matter by blood capillaries.

Author Contributions: Conceptualization: C.C., L.M.-W., and B.M. Methodology: C.C., L.M.-W., R.S., M.S., and B.M. Software: C.C., L.M.-W., and M.S. Writing, original draft: C.C. Writing, review and editing: C.C., L.M.-W., R.S., M.S., J.G., and B.M. Supervision: R.S. and B.M. Funding acquisition: R.S. and B.M. Investigation: J.G. Resources: J.G. Project administration: B.M. All authors have read and agreed to the published version of the manuscript.

Funding: These works were funded by LABEX PRIMES (ANR-11-LABX-0063) of Universite de Lyon, within the program "Investissements d'Avenir" (ANR-11-IDEX-0007), operated by the French National Research Agency (ANR); Cancéropôle Lyon Auvergne Rhône Alpes (CLARA) within the program "OncoStarter"; Infrastructures d'Avenir en Biologie Santé (ANR-11-INBS-000), within the program "Investissements d'Avenir" operated by the French National Research Agency (ANR) and France Life Imaging.

Acknowledgments: We want to acknowledge the PILoT facility for the support provided for the image acquisition.

Conflicts of Interest: No conflicts of interest, financial or otherwise, are declared by the authors.

\section{Abbreviations}

The following abbreviations are used in this manuscript:

$\begin{array}{ll}\mathrm{HbO}_{2} & \text { Oxygenated hemoglobin } \\ \mathrm{Hb} & \text { Deoxygenated hemoglobin } \\ \text { oxCCO } & \text { Oxidative state of cytochrome-c-oxidase } \\ \text { BOLD } & \text { Blood oxygenation level dependent } \\ \text { fMRI } & \text { functional magnetic resonance imaging } \\ \text { NIRS } & \text { Near infrared spectroscopy } \\ \text { SPM } & \text { Statistical parametric mapping } \\ \text { SNR } & \text { Signal-to-noise ratio }\end{array}$

\section{References}

1. Ogawa, S.; Lee, T.M.; Kay, A.R.; Tank, D.W. Brain magnetic resonance imaging with contrast dependent on blood oxygenation. Proc. Natl. Acad. Sci. USA 1990, 87, 9868-9872. [CrossRef]

2. Gerard, I.J.; Kersten-Oertel, M.; Petrecca, K.; Sirhan, D.; Hall, J.A.; Collins, D.L. Brain shift in neuronavigation of brain tumors: A review. Med. Image Anal. 2017, 35, 403-420. [CrossRef] [PubMed]

3. Penfield, W.; Boldrey, E. Somatic motor and sensory representation in the cerebral cortex of man as studied by electrical stimulation. Brain 1937, 60, 389-443. [CrossRef]

4. Caredda, C.; Mahieu-Williame, L.; Sablong, R.; Sdika, M.; Guyotat, J.; Montcel, B. Real Time Intraoperative Functional Brain Mapping Based on RGB Imaging. IRBM 2020, 1213, 1-59. [CrossRef]

5. Caredda, C.; Mahieu-Williame, L.; Sablong, R.; Sdika, M.; Alston, L.; Guyotat, J.; Montcel, B. Intraoperative quantitative functional brain mapping using an RGB camera. Neurophotonics 2019, 6, 1-14. [CrossRef]

6. Karthikeyan, P.; Moradi, S.; Ferdinando, H.; Zhao, Z.; Myllylä, T. Optics Based Label-Free Techniques and Applications in Brain Monitoring. Appl. Sci. 2020, 10, 2196. [CrossRef]

7. Lu, G.; Fei, B. Medical hyperspectral imaging: A review. J. Biomed. Opt. 2014, 19, 1-24. [CrossRef]

8. Arridge, S.R.; Cope, M.; Delpy, D.T. The theoretical basis for the determination of optical pathlengths in tissue: temporal and frequency analysis. Phys. Med. Biol. 1992, 37, 1531-1560. [CrossRef] [PubMed]

9. Kohl-bareis, M.; Ebert, B.; Dreier, J.P.; Leithner, C.; Lindauer, U.; Royl, G. Apparatus for Measuring Blood Parameters. U.S. Patent No. 20120277559, 1 November 2012.

10. Pichette, J.; Laurence, A.; Angulo, L.; Lesage, F.; Bouthillier, A.; Nguyen, D.K.; Leblond, F. Intraoperative video-rate hemodynamic response assessment in human cortex using snapshot hyperspectral optical imaging. Neurophotonics 2016, 3, 045003. [CrossRef]

11. Caredda, C.; Mahieu-Williame, L.; Sablong, R.; Sdika, M.; Guyotat, J.; Montcel, B. Real time intraoperative functional brain mapping using a RGB camera. In Clinical and Preclinical Optical Diagnostics II; Brown, J.Q., van Leeuwen, T.G., Eds.; International Society for Optics and Photonics (SPIE): Washington, DC, USA, 2019; Volume 11073, pp. 17-21. [CrossRef] 
12. Caredda, C.; Mahieu-Williame, L.; Sablong, R.; Sdika, M.; Guyotat, J.; Montcel, B. Pixel-wise modified Beer-Lambert model for intraoperative functional brain mapping. In Clinical and Preclinical Optical Diagnostics II; Brown, J.Q., van Leeuwen, T.G., Eds.; International Society for Optics and Photonics (SPIE): Washington, DC, USA, 2019; Volume 11073, pp. 148-152. [CrossRef]

13. Hassanpour, M.S.; White, B.R.; Eggebrecht, A.T.; Ferradal, S.L.; Snyder, A.Z.; Culver, J.P. Statistical analysis of high density diffuse optical tomography. NeuroImage 2014, 85, 104-116. [CrossRef]

14. Ye, J.; Tak, S.; Jang, K.; Jung, J.; Jang, J. NIRS-SPM: Statistical parametric mapping for near-infrared spectroscopy. NeuroImage 2009, 44, 428-447. [CrossRef] [PubMed]

15. Hillman, E.M.C. Optical brain imaging in vivo: techniques and applications from animal to man. J. Biomed. Opt. 2007, 12, 051402. [CrossRef] [PubMed]

16. Lange, F.; Peyrin, F.; Montcel, B. Broadband time-resolved multi-channel functional near-infrared spectroscopy system to monitor in vivo physiological changes of human brain activity. Appl. Opt. 2018, 57, 6417-6429. [CrossRef] [PubMed]

17. Mottin, S.; Montcel, B.; Guillet de Chatellus, H.; Ramstein, S. Functional white laser imaging to study brain oxygen uncoupling/re-coupling in songbirds. J. Cereb. Blood Flow Metab. Off. J. Int. Soc. Cereb. Blood Flow Metab. 2010, 31, 393-400. [CrossRef]

18. Mottin, S.; Montcel, B.; Chatelus, H.; Ramstein, S.; Vignal, C.; Mathevon, N. CORRIGENDUM: Functional white laser imaging to study brain oxygen uncoupling/re-coupling in songbirds. J. Cereb. Blood Flow Metab. Off. J. Int. Soc. Cereb. Blood Flow Metab. 2011, 31, 1170. [CrossRef]

19. Vignal, C.; Boumans, T.; Montcel, B.; Ramstein, S.; Verhoye, M.; Van Audekerke, J.; Mathevon, N.; Van Der Linden, A.; Mottin, S. Measuring brain hemodynamic changes in a songbird: Responses to hypercapnia measured with functional MRI and near-infrared spectroscopy. Phys. Med. Biol. 2008, 53, 2457-2470. [CrossRef]

20. Montcel, B.; Chabrier, R.; Poulet, P. Time-resolved absorption and haemoglobin concentration difference maps: A method to retrieve depth-related information on cerebral hemodynamics. Opt. Express 2006, 14, 12271-12287. Available online: https:/ / www.osapublishing.org/DirectPDFAccess/D392522C-0D5DA58B-B00C065E3FE3DED9_119770/oe-14-25-12271.pdf?da=1\&id=119770\&seq=0\&mobile=no (accessed on 1 June 2020). [CrossRef]

21. Montcel, B.; Chabrier, R.; Poulet, P. Detection of cortical activation with time-resolved diffuse optical methods. Appl. Opt. 2005, 44, 1942-1947. [CrossRef]

22. Wong-Riley, M.T. Cytochrome oxidase: An endogenous metabolic marker for neuronal activity. Trends Neurosci. 1989, 12, 94-101. [CrossRef]

23. Bale, G.; Elwell, C.E.; Tachtsidis, I. From Jöbsis to the present day: A review of clinical near-infrared spectroscopy measurements of cerebral cytochrome-c-oxidase. J. Biomed. Opt. 2016, 21, 091307. [CrossRef]

24. Giannoni, L.; Lange, F.; Davies, A.L.; Dua, A.; Gustavson, B.; Smith, K.J.; Tachtsidis, I. Hyperspectral Imaging of the Hemodynamic and Metabolic States of the Exposed Cortex: Investigating a Commercial Snapshot Solution. In Oxygen Transport to Tissue XL; Thews, O., LaManna, J.C., Harrison, D.K., Eds.; Springer International Publishing: Cham, Switzerland, 2018; pp. 13-20. [CrossRef]

25. Caredda, C.; Mahieu-Williame, L.; Sablong, R.; Sdika, M.; Guyotat, J.; Montcel, B. Intraoperative functional and metabolic brain mapping using hyperspectral imaging. In Clinical and Translational Neurophotonics 2020; Madsen, S.J., Yang, V.X.D., Thakor, N.V., Eds.; International Society for Optics and Photonics (SPIE): Washington, DC, USA, 2020; Volume 11225, pp. 24-30. [CrossRef]

26. Mori, M.; Chiba, T.; Nakamizo, A.; Kumashiro, R.; Murata, M.; Akahoshi, T.; Tomikawa, M.; Kikkawa, Y.; Yoshimoto, K.; Mizoguchi, M.; et al. Intraoperative visualization of cerebral oxygenation using hyperspectral image data: a two-dimensional mapping method. Int. J. Comput. Assist. Radiol. Surg. 2014, 9, 1059-1072. [CrossRef]

27. Arifler, D.; Zhu, T.; Madaan, S.; Tachtsidis, I. Optimal wavelength combinations for near-infrared spectroscopic monitoring of changes in brain tissue hemoglobin and cytochrome c oxidase concentrations. Biomed. Opt. Express 2015, 6, 933-947. [CrossRef]

28. Giannoni, L.; Lange, F.; Tachtsidis, I. Investigation of the quantification of hemoglobin and cytochrome-c-oxidase in the exposed cortex with near-infrared hyperspectral imaging: A simulation study. J. Biomed. Opt. 2020, 25, 1-25. [CrossRef] [PubMed] 
29. Sudakou, A.; Wojtkiewicz, S.; Lange, F.; Gerega, A.; Sawosz, P.; Tachtsidis, I.; Liebert, A. Depth-resolved assessment of changes in concentration of chromophores using time-resolved near-infrared spectroscopy: estimation of cytochrome-c-oxidase uncertainty by Monte Carlo simulations. Biomed. Opt. Express 2019, 10, 4621-4635. [CrossRef]

30. Chen, L.Y.; Pan, M.C.; Yan, C.C.; Pan, M.C. Wavelength optimization using available laser diodes in spectral near-infrared optical tomography. Appl. Opt. 2016, 55, 5729-5737. [CrossRef] [PubMed]

31. Ocean Optics. USB 2000 Spectrometer. Available online: www.ugastro.berkeley.edu (accessed on 1 June 2020).

32. Bayer, B.E. Color Imaging Array. U.S. Patent No. 3971065 A, 20 July 1976. p. 10.

33. Benoit, L.; Benoit, R.; Belin, E.; Vadaine, R.; Demilly, D.; Chapeau-Blondeau, F.; Rousseau, D. On the value of the Kullback-Leibler divergence for cost-effective spectral imaging of plants by optimal selection of wavebands. Mach. Vis. Appl. 2016, 27, 625-635. [CrossRef]

34. Fang, Q.; Boas, D.A. Monte Carlo simulation of photon migration in 3D turbid media accelerated by graphics processing units. Opt. Express 2009, 17, 20178-20190. [CrossRef]

35. Schotland, J.C.; Haselgrove, J.C.; Leigh, J.S. Photon hitting density. Appl. Opt. 1993, 32, 448-453. [CrossRef] [PubMed]

36. Mitchell, H.H.; Hamilton, T.S.; Steggerda, F.R.; Bean, H.W. The chemical composition of the adult human body and its bearing on the biochemistry of growth. J. Biol. Chem. 1945, 158, 625-637. Available online: https://www.jbc.org/content/158/3/625.full.pdf (accessed on 1 June 2020).

37. Gagnon, L.; Gauthier, C.; Hoge, R.D.; Lesage, F.; Selb, J.; Boas, D.A. Double-layer estimation of intra- and extracerebral hemoglobin concentration with a time-resolved system. J. Biomed. Opt. 2008, 13, 054019. [CrossRef]

38. Binding, J.; Ben Arous, J.; Léger, J.F.; Gigan, S.; Boccara, C.; Bourdieu, L. Brain refractive index measured in vivo with high-NA defocus-corrected full-field OCT and consequences for two-photon microscopy. Opt. Express 2011, 19, 4833. [CrossRef] [PubMed]

39. Jacques, S.L. Optical properties of biological tissues: A review. Phys. Med. Biol. 2013, 58, R37-R61. [CrossRef] [PubMed]

40. Cope, M. The Application of Near Infrared Spectroscopy to Non Invasive Monitoring of Cerebral Oxygenation in the Newborn Infant. Ph.D. Thesis, University College London, London, UK, 1991.

41. Mason, M.G.; Nicholls, P.; Cooper, C.E. Re-evaluation of the near-infrared spectra of mitochondrial cytochrome c oxidase: Implications for non invasive in vivo monitoring of tissues. Biochim. Biophys. Acta Bioenerg 2014, 1837, 1882-1891. [CrossRef]

42. Yaroslavsky, A.N.; Schulze, P.C.; Yaroslavsky, I.V.; Schober, R.; Ulrich, F.; Schwarzmaier, H.J. Optical properties of selected native and coagulated human brain tissues in vitro in the visible and near-infrared spectral range. Phys. Med. Biol. 2002, 47, 2059-2073. [CrossRef] [PubMed]

43. Steimers, A.; Gramer, M.; Ebert, B.; Füchtemeier, M.; Royl, G.; Leithner, C.; Dreier, J.P.; Lindauer, U.; Kohl-Bareis, M. Imaging of Cortical Haemoglobin Concentration with RGB Reflectometry; Optical Society of America: Washington, DC, USA, 2009; p. 736813. [CrossRef]

44. Delpy, D.T.; Cope, M.; Zee, P.v.d.; Arridge, S.; Wray, S.; Wyatt, J. Estimation of optical pathlength through tissue from direct time of flight measurement. Phys. Med. Biol. 1988, 33, 1433-1442. [CrossRef] [PubMed]

45. Wobst, P.; Wenzel, R.; Kohl, M.; Obrig, H.; Villringer, A. Linear Aspects of Changes in Deoxygenated Hemoglobin Concentration and Cytochrome Oxidase Oxidation during Brain Activation. NeuroImage 2001, 13, 520-530. [CrossRef]

46. Matcher, S.; Elwell, C.; Cooper, C.; Cope, M.; Delpy, D. Performance Comparison of Several Published Tissue Near-Infrared Spectroscopy Algorithms. Anal. Biochem. 1995, 227, 54-68. [CrossRef] [PubMed]

47. Culver, J.P.; Siegel, A.M.; Franceschini, M.A.; Mandeville, J.B.; Boas, D.A. Evidence that cerebral blood volume can provide brain activation maps with better spatial resolution than deoxygenated hemoglobin. NeuroImage 2005, 27, 947-959. [CrossRef] [PubMed]

(C) 2020 by the authors. Licensee MDPI, Basel, Switzerland. This article is an open access article distributed under the terms and conditions of the Creative Commons Attribution (CC BY) license (http:/ / creativecommons.org/licenses/by/4.0/). 